\title{
Learning a Strategy for Adapting a Program Analysis via Bayesian Optimisation
}

\author{
Hakjoo Oh \\ Korea University \\ hakjoo.oh@gmail.com
}

\author{
Hongseok Yang \\ University of Oxford \\ hongseok.yang@cs.ox.ac.uk
}

\author{
Kwangkeun Yi \\ Seoul National University \\ kwang@ropas.snu.ac.kr
}

\begin{abstract}
Building a cost-effective static analyser for real-world programs is still regarded an art. One key contributor to this grim reputation is the difficulty in balancing the cost and the precision of an analyser. An ideal analyser should be adaptive to a given analysis task, and avoid using techniques that unnecessarily improve precision and increase analysis cost. However, achieving this ideal is highly nontrivial, and it requires a large amount of engineering efforts.

In this paper we present a new approach for building an adaptive static analyser. In our approach, the analyser includes a sophisticated parameterised strategy that decides, for each part of a given program, whether to apply a precision-improving technique to that part or not. We present a method for learning a good parameter for such a strategy from an existing codebase via Bayesian optimisation. The learnt strategy is then used for new, unseen programs. Using our approach, we developed partially flowand context-sensitive variants of a realistic $\mathrm{C}$ static analyser. The experimental results demonstrate that using Bayesian optimisation is crucial for learning from an existing codebase. Also, they show that among all program queries that require flow- or context-sensitivity, our partially flow- and context-sensitive analysis answers the $75 \%$ of them, while increasing the analysis cost only by $3.3 \mathrm{x}$ of the baseline flow- and context-insensitive analysis, rather than $40 \mathrm{x}$ or more of the fully sensitive version.
\end{abstract}

Categories and Subject Descriptors F.3.2 [Semantics of Programming Languages]: Program Analysis; G.1.6 [Optimization]: Global Optimization; D.2.4 [Software/Program Verification]: Assertion Checkers; I.2.6 [Learning]: Parameter Learning
Keywords Program Analysis, Bayesian Optimization

\section{Introduction}

Although the area of static program analysis has progressed substantially in the past two decades, building a cost-effective static analyser for real-world programs is still regarded an art. One key contributor to this grim reputation is the difficulty in balancing the cost and the precision of an analyser. An ideal analyser should be able to adapt to a given analysis task automatically, and avoid using techniques that unnecessarily improve precision and increase analysis cost. However, designing a good adaptation strategy is highly nontrivial, and it requires a large amount of engineering efforts.

In this paper we present a new approach for building an adaptive static analyser, which can learn its adaptation strategy from an existing codebase. In our approach, the analyser includes a parameterised strategy that decides, for each part of a given program, whether to apply a precision-improving technique to that part or not. This strategy is defined in terms of a function that scores parts of a program. The strategy evaluates parts of a given program using this function, chooses the top $k$ parts for some fixed $k$, and applies the precision-improving technique to these parts only. The parameter of the strategy controls this entire selection process by being a central component of the scoring function.

Of course, the success of such an analyser depends on finding a good parameter for its adaptation strategy. We describe a method for learning such a parameter from an existing codebase using Bayesian optimisation; the learnt parameter is then used for new, unseen programs. As typical in other machine learning techniques, this learning part is formulated as an optimisation problem: find a parameter that maximises the number of queries in the codebase which are proved by the analyser. This is a challenging optimisation problem because evaluating its objective function involves running the analyser over several programs and so it is expensive. We present an (approximate) solver for the problem that uses the powerful Bayesian optimisation technique and avoids expensive calls to the program analyser as much as possible. 
Using our approach, we developed partially flow-sensitive and context-sensitive variants of a realistic $\mathrm{C}$ program analyser. The experimental results confirm that using an efficient optimisation solver such as ours based on Bayesian optimisation is crucial for learning a good parameter from an existing codebase; a naive approach for learning simply does not scale. When our partially flow- and context-sensitive analyser was run with a learnt parameter, it answered the $75 \%$ of the program queries that require flow- or context-sensitivity, while increasing the analysis cost only by $3.3 x$ of the flowand context-insensitive analysis, rather than 40x or more of the fully sensitive version.

Contributions We summarise our contributions below:

- We propose a new approach for building a program analysis that can adapt to a given verification task. The key feature of our approach is that it can learn an adaptation strategy from an existing codebase automatically, which can then be applied to new unseen programs.

- We present an effective method for learning an adaptation strategy. Our method uses powerful Bayesian optimisation techniques, and reduces the number of expensive program-analysis runs on given programs during the learning process. The performance gain by Bayesian optimisation is critical for making our approach practical; without it, learning with medium-to-large programs takes too much time.

- We describe two instance analyses of our approach, which are adaptive variants of our program analyser for $\mathrm{C}$ programs. The first adapts the degree of flow sensitivity of the analyser, and the second, that of both flow and context sensitivities of the analyser. Our experiments show the clear benefits of our approach.

\section{Overview}

We illustrate our approach using a static analysis with the interval domain. Consider the following program.

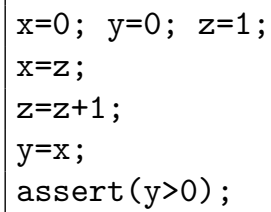

The program has three variables $(x, y$, and $z)$ and the goal of the analysis is to prove that the assertion at line 5 holds.

\subsection{Partially flow-sensitive analysis}

Our illustration uses a partially flow-sensitive analysis. Given a set of variables $V$, it tracks the values of selected variables in $V$ flow-sensitively, but for the other variables, it computes global flow-insensitive invariants of their values. For instance, when $V=\{x, y\}$, the analysis computes the following results:

\begin{tabular}{|c|l|c|}
\hline \multicolumn{2}{|c|}{ flow-sensitive } & flow-insensitive \\
\hline line & \multicolumn{1}{|c|}{ abstract state } & abstract state \\
\hline 1 & $\{x \mapsto[0,0], \quad y \mapsto[0,0]\}$ & \\
2 & $\{x \mapsto[1,+\infty], y \mapsto[0,0]\}$ & \\
3 & $\{x \mapsto[1,+\infty], y \mapsto[0,0]\}$ & $\{z \mapsto[1,+\infty]\}$ \\
4 & $\{x \mapsto[1,+\infty], y \mapsto[1,+\infty]\}$ & \\
5 & $\{x \mapsto[1,+\infty], y \mapsto[1,+\infty]\}$ & \\
\hline
\end{tabular}

The results are divided into two parts: flow-sensitive and flow-insensitive results. In the flow-sensitive part, the analysis maintains an abstract state at each program point, where each state involves only the variables in $V$. The information for the other variables $(z)$ is kept in the flow-insensitive state, which is a single abstract state valid for the entire program. Note that this partially flow-sensitive analysis is precise enough to prove the given assertion; at line 5, the analysis concludes that $y$ is greater than 0 .

In our example, our $\{x, y\}$ and the entire set $\{x, y, z\}$ are the only choices of $V$ that lead to the proof of the assertion: with any other choice $(V \in\{\emptyset,\{x\},\{y\},\{z\},\{x, z\},\{y, z\}\})$, the analysis fails to prove the assertion. Our analysis adapts to the program here automatically and picks $V$. We will next explain how this adaption happens.

\subsection{Adaptation strategy parameterised with w}

Our analysis employs a parameterised strategy (or decision rule) for selecting a set $V$ of variables that will be treated flow-sensitively. The strategy is a function of the form:

$$
\mathcal{S}_{\mathbf{w}}: P g m \rightarrow \wp(\text { Var })
$$

which is parameterised by a vector $\mathbf{w}$ of real numbers.

Given a program to analyse, our strategy works in three steps:

1. We represent all the variables of the program as feature vectors.

2. We then compute the score of each variable $x$, which is just the linear combination of the parameter $\mathbf{w}$ and the feature vector of $x$.

3. We choose the top- $k$ variables based on their scores, where $k$ is specified by users. In this example, we use $k=2$.

Step 1: Extracting features Our analysis uses a preselected set $\pi$ of features, which are just predicates on variables and summarise syntactic or semantic properties of variables in a given program. For instance, a feature $\pi_{i} \in \pi$ indicates whether a variable is a local variable of a function or not. These feature predicates are chosen for the analysis, and reused for all programs. The details of the features that we used are given in later sections of this paper. In the example of this section, let us assume that our feature set $\pi$ consists of five predicates:

$$
\pi=\left\{\pi_{1}, \pi_{2}, \pi_{3}, \pi_{4}, \pi_{5}\right\} .
$$


Given a program and a feature set $\pi$, we can represent each variable $x$ in the program as a feature vector $\pi(x)$ :

$$
\pi(x)=\left\langle\pi_{1}(x), \pi_{2}(x), \pi_{3}(x), \pi_{4}(x), \pi_{5}(x)\right\rangle
$$

Suppose that the feature vectors of variables in the example program are as follows:

$$
\begin{aligned}
& \pi(x)=\langle 1,0,1,0,0\rangle \\
& \pi(y)=\langle 1,0,1,0,1\rangle \\
& \pi(z)=\langle 0,0,1,1,0\rangle
\end{aligned}
$$

Step 2: Scoring Next, we computes the scores of variables based on the feature vectors and the parameter $\mathbf{w}$. The parameter $\mathbf{w}$ is a real-valued vector that has the same dimension as the feature vector, i.e., in this example, $\mathbf{w} \in \mathbb{R}^{5}$ for $\mathbb{R}=[-1,1]$. Intuitively, $\mathbf{w}$ encodes the relative importance of each feature.

Given a parameter $\mathbf{w} \in \mathbb{R}^{5}$, e.g.,

$$
\mathbf{w}=\langle 0.9,0.5,-0.6,0.7,0.3\rangle
$$

the score of variable $x$ is computed as follows:

$$
\operatorname{score}(x)=\pi(x) \cdot \mathbf{w}
$$

In our example, the scores of $x, y$, and $z$ are:

$$
\begin{aligned}
& \text { score }(x)=\langle 1,0,1,0,0\rangle \cdot\langle 0.9,0.5,-0.6,0.7,0.3\rangle=0.3 \\
& \text { score }(y)=\langle 1,0,1,0,1\rangle \cdot\langle 0.9,0.5,-0.6,0.7,0.3\rangle=0.6 \\
& \operatorname{score}(z)=\langle 0,0,1,1,0\rangle \cdot\langle 0.9,0.5,-0.6,0.7,0.3\rangle=0.1
\end{aligned}
$$

Step 3: Choosing top- $k$ variables Finally, we choose the top- $k$ variables based on their scores. For instance, when $k=$ 2 , we choose variables $x$ and $y$ in our example. As we have already pointed out, this is the right choice in our example because analysing the example program with $V=\{x, y\}$ proves the assertion.

\subsection{Learning the parameter $\mathrm{w}$}

Finding a good parameter $\mathbf{w}$ manually is difficult. We expect that a program analysis based on our approach uses more than 30 features, so its parameter $\mathbf{w}$ lives in $\mathbb{R}^{n}$ for some $n \geq 30$. This is a huge search space. It is unrealistic to ask a human to acquire intuition on this space and come up with a right $\mathbf{w}$ that leads to a suitable adaptation strategy for most programs in practice. ${ }^{1}$

The learning part of our approach aims at finding a good w automatically. It takes a codebase consisting of typical programs, and searches for a parameter $\mathbf{w}$ that instantiates an adaptation strategy appropriately for programs in the codebase: with this instantiation, a program analysis can prove a large number of queries in these programs.

\footnotetext{
${ }^{1}$ In our experiments, all manually chosen parameters lead to strategies that perform much worse than the one automatically learnt by the method in this subsection.
}

We explain how our learning algorithm works by using a small codebase that consists of just the following two programs:

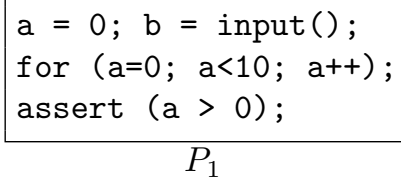

Given this codebase, our learning algorithm looks for $\mathbf{w}$ that makes the analysis prove the two assert statements in $P_{1}$ and $P_{2}$. Our intention is to use the learnt $\mathbf{w}$ later when analysing new unseen programs (such as the example in the beginning of this section). We assume that program variables in $P_{1}$ and $P_{2}$ are summarised by feature vectors as follows:

$$
\begin{aligned}
\pi(a) & =\langle 0,1,1,0,1\rangle, \quad \pi(b)=\langle 1,0,0,1,0\rangle \\
\pi(c) & =\langle 0,1,0,0,1\rangle, \quad \pi(d)=\langle 1,1,0,1,0\rangle
\end{aligned}
$$

Simple algorithm based on random sampling Let us start with a simple learning algorithm that uses random sampling. Going through this simple algorithm will help a reader to understand our learning algorithm based on Bayesian optimisation. The algorithm based on random sampling works in four steps. Firstly, it generates $n$ random samples in the space $\mathbb{R}^{5}$. Secondly, for each sampled parameter $\mathbf{w}_{i} \in \mathbb{R}^{5}$, the algorithm instantiates the strategy with $\mathbf{w}_{i}$, runs the static analysis with the variables chosen by the strategy, and records how many assertions in the given codebase are proved. Finally, it chooses the parameter $\mathbf{w}_{i}$ with the highest number of proved assertions.

The following table shows the results of running this algorithm on our codebase $\left\{P_{1}, P_{2}\right\}$ with $n=5$. For each sampled parameter $\mathbf{w}_{i}$, the table shows the variables selected by the instantiated strategy with $\mathbf{w}_{i}$ (here we assume that we choose $k=1$ variable from each program), and the number of assertions proved in the codebase.

\begin{tabular}{|c|c|c|c|c|c|}
\hline try & sample $\mathbf{w}_{i}$ & \multicolumn{2}{|c|}{ decision } & \multicolumn{2}{|c|}{ \#proved } \\
\cline { 3 - 6 } & & $P_{1}$ & $P_{2}$ & $P_{1}$ & $P_{2}$ \\
\hline 1 & $-0.0,0.7,-0.9,1.0,-0.7$ & $\mathrm{~b}$ & $\mathrm{~d}$ & 0 & 1 \\
2 & $0.2,-0.0,-0.8,-0.5,-0.2$ & $\mathrm{~b}$ & $\mathrm{c}$ & 0 & 0 \\
3 & $0.4,-0.6,-0.6,0.6,-0.7$ & $\mathrm{~b}$ & $\mathrm{~d}$ & 0 & 1 \\
4 & $-0.5,0.5,-0.5,-0.6,-0.9$ & $\mathrm{a}$ & $\mathrm{c}$ & 1 & 0 \\
5 & $-0.6,-0.8,-0.1,-0.9,-0.2$ & $\mathrm{a}$ & $\mathrm{c}$ & 1 & 0 \\
\hline
\end{tabular}

Four parameters achieve the best result, which is to prove one assert statement (either from $P_{1}$ or $P_{2}$ ). Among these four, the algorithm returns one of them, such as:

$$
\mathbf{w}=\langle-0.0,-0.7,0.9,1.0,-0.7\rangle .
$$

Note that this is not an ideal outcome; we would like to prove both assert statements. In order to achieve this ideal, our analysis needs to select variables $a$ from $P_{1}$ and $d$ from $P_{2}$ and treat them flow-sensitively. But random searching has low probability for finding $\mathbf{w}$ that leads to this variable 


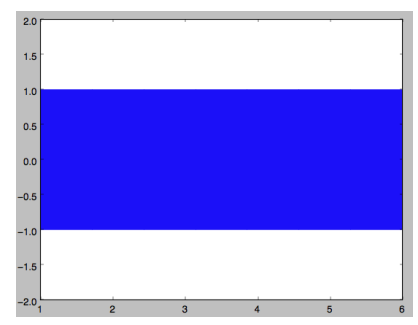

(a)

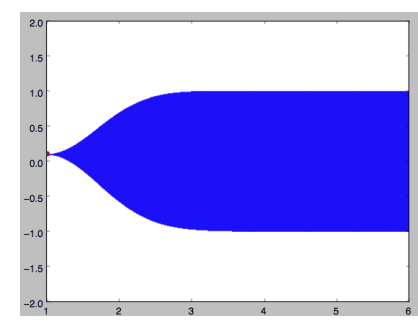

(b)

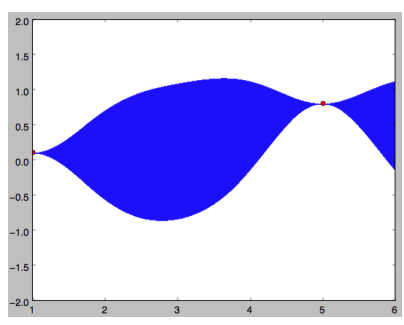

(c)

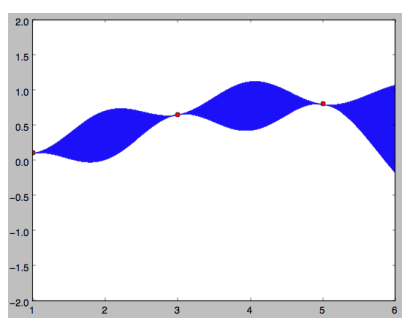

(d)

Figure 1. A typical scenario on how the probabilistic model gets updated during Bayesian optimisation.

selection. This shortcoming of random sampling is in a sense expected, and it does appear in practice. As we show in Figure 2, most of the randomly sampled parameters in our experiments perform poorly. Thus, in order to find a good parameter via random sampling, we need a large number of trials, but each trial is expensive because it involves running a static analysis over all the programs in a given codebase.

Bayesian optimisation To describe our algorithm, we need to be more precise about the setting and the objective of algorithms for learning $\mathbf{w}$. These learning algorithms treat a program analysis and a given codebase simply as a specification of an (objective) function

$$
F: \mathbb{R}^{n} \rightarrow \mathbb{N} .
$$

The input to the function is a parameter $\mathbf{w} \in \mathbb{R}^{n}$, and the output is the number of queries in the codebase that are proved by the analysis. The objective of the learning algorithms is to find $\mathbf{w}^{*} \in \mathbb{R}^{n}$ that maximises the function $F$ :

$$
\text { Find } \mathbf{w}^{*} \in \mathbb{R}^{n} \text { that maximises } F(\mathbf{w}) \text {. }
$$

Bayesian optimisation $[3,15]$ is a generic algorithm for solving an optimisation where an objective function does not have a nice mathematical structure such as gradient and convexity, and evaluating this function is expensive. It aims at minimising the evaluation of the objective function as much as possible. Notice that our objective function $F$ in (2) lacks good mathematical structures and is expensive to evaluate, so it is a good target of Bayesian optimisation. Also, the aim of Bayesian optimisation is directly related to the inefficiency of the random-sampling algorithm mentioned above.

The basic structure of Bayesian optimisation is similar to the random sampling algorithm. It repeatedly evaluates the objective function with different inputs until it reaches a time limit, and returns the best input found. However, Bayesian optimisation diverges from random sampling in one crucial aspect: it builds a probability model about the objective function, and uses the model for deciding where to evaluate the function next. Bayesian optimisation builds the model based on the results of the evaluation so far, and updates the model constantly according to the standard rules of Bayesian statistics when it evaluates the objective function with new inputs.
In the rest of this overview, we focus on explaining informally how typical Bayesian optimisation builds and uses a probabilistic model, instead of specifics of our algorithm. This will help a reader to see the benefits of Bayesian optimisation in our problem. The full description of our learning algorithm is given in Section 5.3.

Assume that we are given an objective function $G$ of type $\mathbb{R} \rightarrow \mathbb{R}$. Bayesian optimisation constructs a probabilistic model for this unknown function $G$, where the model expresses the optimiser's current belief about $G$. The model defines a distribution on functions of type $\mathbb{R} \rightarrow \mathbb{R}$ (using so called Gaussian process [21]). Initially, it has high uncertainty about what $G$ is, and assumes that positive outputs or negative outputs are equally possible for $G$, so the mean (i.e., average) of this distribution is the constant zero function $\lambda x .0$. This model is shown in Figure 1(a), where the large blue region covers typical functions sampled from this model.

Suppose that Bayesian optimisation chooses $x=1.0$, evaluates $G(1.0)$, and get 0.1 as the output. Then, it incorporates this input-output pair, $(1.0,0.1)$, for $G$ into the model. The updated model is shown in Figure 1(b). It now says that $G(1.0)$ is definitely 0.1 , and that evaluating $G$ near 1.0 is likely to give an output similar to 0.1 . But it remains uncertain about $G$ at inputs further from 1.0.

Bayesian optimisation uses the updated model to decide a next input to use for evaluation. This decision is based on balancing two factors: one for exploiting the model and finding the maximum of $G$ (called exploitation), and the other for evaluating $G$ with an input very different from old ones and refining the model based on the result of this evaluation (called exploration). This balancing act is designed so as to minimise the number of evaluation of the objective function. For instance, Bayesian optimisation now may pick $x=5.0$ as the next input to try, because the model is highly uncertain about $G$ at this input. If the evaluation $G(5.0)$ gives 0.8 , Bayesian optimisation updates the model to one in in Figure 1. Next, Bayesian optimisation may decide to use the input $x=3.0$ because the model predicts that $G$ 's output at 3.0 reasonably high on average but it has high uncertainty around this input. If $G(3.0)=0.65$, Bayesian optimisation updates the model as shown in Figure 1(d). At this point, Bayesian optimisation may decide that exploiting the model 
so far outweighs the benefit of exploring $G$ with new inputs, and pick $x=4.0$ where $G$ is expected to give a high value according to the model. By incorporating all the information about $G$ into the model and balancing exploration and exploitation, Bayesian optimisation fully exploits all the available knowledge about $G$, and minimises the expensive evaluation of the function $G$.

\section{Adaptive static analysis}

We use a well-known setup for building an adaptive (or parametric) program analysis [12]. In this approach, an analysis has switches for parts of a given program that determine whether these parts should be analysed with high precision or not. It adapts to the program by turning on these switches selectively according to a fixed strategy. ${ }^{2}$ For instance, a partially context-sensitive analysis has switches for call sites of a given program, and use them to select call sites that will be treated with context sensitivity.

Let $P \in \mathbb{P}$ be a program that we would like to analyse. We assume a set $\mathbb{J}_{P}$ of indices that represent parts of $P$. We define a set of abstractions as follows:

$$
\mathbf{a} \in \mathcal{A}_{P}=\{0,1\}^{\mathbb{J}_{P}},
$$

Abstractions are binary vectors with indices in $\mathbb{J}_{P}$, and are ordered pointwise:

$$
\mathbf{a} \sqsubseteq \mathbf{a}^{\prime} \Longleftrightarrow \forall j \in \mathbb{J}_{P} \cdot \mathbf{a}_{j} \leq \mathbf{a}^{\prime}{ }_{j}
$$

Intuitively, $\mathbb{J}_{P}$ consists of the parts of $P$ where we have switches for controlling the precision of an analysis. For instance, in a partially context-sensitive analysis, $\mathbb{J}_{P}$ is the set of procedures or call sites in the program. In our partially flow-sensitive analysis, it denotes the set of program variables that are analysed flow-sensitively. An abstraction a is just a particular setting of the switches associated with $\mathbb{J}_{P}$, and determines a program abstraction to be used by the analyser. Thus, $\mathbf{a}_{j}=1$ means that the component $j \in \mathbb{J}_{P}$ is analysed, e.g., with context sensitivity or flow sensitivity. We sometimes regard an abstraction $\mathbf{a} \in \mathcal{A}_{P}$ as a function from $\mathbb{J}_{P}$ to $\{0,1\}$, or the following collection of $P$ 's parts:

$$
\mathbf{a}=\left\{j \in \mathbb{J}_{P} \mid \mathbf{a}_{j}=1\right\} .
$$

In the latter case, we write $|\mathbf{a}|$ for the size of the collection. The last bit of notations is two constants in $\mathcal{A}_{P}$ :

$$
\mathbf{0}=\lambda j \in \mathbb{J}_{P .0}, \quad \text { and } \quad \mathbf{1}=\lambda j \in \mathbb{J}_{P .1},
$$

which represent the most imprecise and precise abstractions, respectively. In the rest of this paper, we omit the subscript $P$ when there is no confusion.

\footnotetext{
2 This type of an analysis is usually called parametric program analysis. We do not use this phrase in the paper to avoid confusion; if we did, we would have two types of parameters, ones for selecting parts of a given program, and the others for deciding a particular adaption strategy of the analysis.
}

We assume that a set of assertions is given together with $P$. The goal of the analysis is to prove as many assertions as possible. An adaptive static analysis is modelled as a function:

$$
F: P g m \times \mathcal{A} \rightarrow \mathbb{N}
$$

Given an abstraction $\mathbf{a} \in \mathcal{A}, F(P, \mathbf{a})$ returns the number of assertions proved under the abstraction a. Usually, the used abstraction correlates the precision and the performance of the analysis. That is, using a more refined abstraction is likely to improve the precision of the analysis but increase its cost. ${ }^{3}$ Thus, most existing adaptation strategies aim at finding a small a that makes the analysis prove as many queries as the abstraction $\mathbf{1}$.

\subsection{Goal}

Our goal is to learn a good adaptation strategy automatically from an existing codebase $\mathbf{P}=\left\{P_{1}, \ldots, P_{m}\right\}$ (that is, a collection of programs). A learnt strategy is a function of the following type: ${ }^{4}$

$$
\mathcal{S}: P g m \rightarrow \mathcal{A}
$$

and is used to analyse new, unseen programs $P$;

$$
F(P, \mathcal{S}(P)) \text {. }
$$

If the learnt strategy is good, running the analysis with $\mathcal{S}(P)$ would give results close to those of the most precise abstraction $(F(P, \mathbf{1}))$, while incurring the cost at the level of or only slightly above the least precise and hence cheapest abstraction $(F(P, \mathbf{0}))$.

In order to achieve our goal, we need to address two well-known challenges from the machine learning literature. First, our learning algorithm should be able to generalise its findings from a given codebase $\mathbf{P}$, and derive an adaptation strategy that works well for unseen programs, or at least those similar to the programs in $\mathbf{P}$. In our setting, this means that we need to identify a restricted space $\mathcal{H} \subseteq[\mathrm{Pgm} \rightarrow \mathcal{A}]$ of adaptation strategies, called hypothesis space, such that solving the following optimisation problem over the given $\mathbf{P}$ gives a good strategy for unseen programs $P$ :

$$
\text { Find } \mathcal{S} \in \mathcal{H} \text { that maximises } \sum_{P_{i} \in \mathbf{P}} F\left(P_{i}, \mathcal{S}\left(P_{i}\right)\right) \text {. }
$$

Here a good strategy should meet both precision and accuracy criteria. Intuitively, our task here is to find a hypothesis space $\mathcal{H}$ of strategies that are based on general adaptation principles, not ad-hoc program-specific properties, so that optimisation above does not lead to a strategy overfit to $\mathbf{P}$.

\footnotetext{
${ }^{3}$ If $\mathbf{a} \sqsubseteq \mathbf{a}^{\prime}$, we typically have $F(P, \mathbf{a}) \leq F\left(P, \mathbf{a}^{\prime}\right)$, but performing $F\left(P, \mathbf{a}^{\prime}\right)$ costs more than performing $F(P, \mathbf{a})$.

${ }^{4}$ Strictly speaking, the set of abstractions varies depending on a given program, so a strategy is a dependently-typed function and maps a program to one of the abstractions associated with the program. We elide this distinction to simplify presentation.
} 
Second, we need an efficient method for solving the optimisation problem in (3). Note that evaluating the objective function of (3) involves running the static analysis on all programs in $\mathbf{P}$, which is very expensive. Thus, while solving the optimisation problem, we need to avoid the evaluation of the objective function as much as possible.

In the rest of the paper, we explain how we address these challenges. For the first challenge, we define a hypothesis space $\mathcal{H}$ of parameterised adaptation strategies that score program parts based on parameterised linear functions and select high scorers for receiving precise analysis (Section 4). For the second challenge, we use Bayesian optimisation, which attempts to minimise the evaluation of an objective function by building a Bayesian model about this function (Section 5).

\section{Parameterised adaptation strategy}

In this section, we explain a parameterised adaptation strategy, which defines our hypothesis space $\mathcal{H}$ mentioned in the previous section. Intuitively, this parameterised adaptation strategy is a template for all the candidate strategies that our analysis can use when analysing a given program, and its instantiations with different parameter values form $\mathcal{H}$.

Recall that for a given program $P$, an adaption strategy chooses a set of components of $P$ that will be analysed with high precision. As explained in Section 2.2, our parameterised strategy makes this choice in three steps. We formalize these steps next.

\subsection{Feature extraction}

Given a program $P$, our parameterised strategy first represents $P$ 's components by so called feature vectors. A feature $\pi^{k}$ is a predicate on program components:

$$
\pi_{P}^{k}: \mathbb{J}_{P} \rightarrow\{0,1\} \text { for each program } P .
$$

For instance, when components in $\mathbb{J}_{P}$ are program variables, checking whether a variable $j$ is a local variable or not is a feature. Our parameterised strategy requires that a static analysis comes with a collection of features:

$$
\pi_{P}=\left\{\pi_{P}^{1}, \ldots, \pi_{P}^{n}\right\} .
$$

Using these features, the strategy represents each program component $j$ in $\mathbb{J}_{P}$ as a boolean vector as follows:

$$
\pi_{P}(j)=\left\langle\pi_{P}^{1}(j), \ldots, \pi_{P}^{n}(j)\right\rangle .
$$

We emphasise that the same set of features is reused for all programs, as long as the same static analysis is applied to them.

As in any other machine learning approaches, choosing a good set of features is critical for the effectiveness of our learning-based approach. We discuss our choice of features for two instance program analyses in Section 6. According to our experience, finding these features required efforts, but was not difficult, because the used features were mostly well-known syntactic properties of program components.

\subsection{Scoring}

Next, our strategy computes the scores of program components using a linear function of feature vectors: for a program $P$,

$$
\begin{gathered}
\operatorname{score}_{P}^{\mathbf{w}}: \mathbb{J}_{P} \rightarrow \mathbb{R} \\
\operatorname{score}_{P}^{\mathbf{w}}(j)=\pi_{P}(j) \cdot \mathbf{w} .
\end{gathered}
$$

Here we assume $\mathbb{R}=[-1,1]$ and $\mathbf{w} \in \mathbb{R}^{n}$ is a real-valued vector with the same dimension as the feature vector. The vector $\mathbf{w}$ is the parameter of our strategy, and determines the relative importance of each feature when our strategy chooses a set of program components.

We extend the score function to abstractions a:

$$
\operatorname{score}_{P}^{\mathbf{w}}(\mathbf{a})=\sum_{j \in \mathbb{J}_{P} \wedge \mathbf{a}(j)=1} \operatorname{score}_{P}^{\mathbf{w}}(j),
$$

which sums the scores of the components chosen by $\mathbf{a}$.

\subsection{Selecting top- $k$ components}

Finally, our strategy selects program components based on their scores, and picks an abstraction accordingly. Given a fixed $k \in \mathbb{R}(0 \leq k \leq 1)$, it chooses $\left\lfloor k \times\left|\mathbb{J}_{P}\right|\right\rfloor$ components with highest scores. For instance, when $k=0.1$, it chooses the top $10 \%$ of program components. Then, the strategy returns an abstraction a that maps these chosen components to 1 and the rest to 0 .

Let $\mathcal{A}^{k}$ be the set of abstractions that contains $\left\lfloor k \times\left|\mathbb{J}_{P}\right|\right\rfloor$ elements when viewed as a set of $j \in \mathbb{J}_{P}$ with $\mathbf{a}_{j}=1$ :

$$
\mathcal{A}^{k}=\left\{\mathbf{a} \in \mathcal{A}|| \mathbf{a} \mid=\left\lfloor k \times\left|\mathbb{J}_{P}\right|\right\rfloor\right\}
$$

Formally, our parameterised strategy $\mathcal{S}_{\mathbf{w}}: \operatorname{Pgm} \rightarrow \mathcal{A}^{k}$ is defined as follows:

$$
\mathcal{S}_{\mathbf{w}}(P)=\underset{\mathbf{a} \in \mathcal{A}_{P}^{k}}{\operatorname{argmax}} \operatorname{score}_{P}^{\mathbf{w}}(\mathbf{a})
$$

That is, given a program $P$ and a parameter $\mathbf{w}$, it selects an abstraction $\mathbf{a} \in \mathcal{A}^{k}$ with maximum score.

A reader might wonder which $k$ value should be used. In our case, we set $k$ close to 0 (e.g. $k=0.1$ ) so that our strategy choose a small and cheap abstraction. Typically, this in turn entails a good performance of the analysis with the chosen abstraction.

Using such a small $k$ is based on a conjecture that for many verification problems, the sizes of minimal abstractions sufficient for proving these problems are significantly small. One evidence of this conjecture is given by Liang and Naik [12], who presented algorithms to find minimal abstractions (the coarsest abstraction sufficient to prove all the queries provable by the most precise abstraction) and showed that, in a pointer analysis used for discharging queries from a race detector, only a small fraction (0.4$2.3 \%$ ) of call-sites are needed to prove all the queries prov- 


\begin{tabular}{|l|r|r|r|r|r|r|r|}
\hline Program & \#Var & \multicolumn{2}{|c|}{ Flow-insensitivity } & \multicolumn{2}{|c|}{ Flow-sensitivity } & \multicolumn{2}{c|}{ Minimal flow-sensitivity } \\
\cline { 3 - 7 } & & proved & time(s) & proved & time(s) & time(s) & size \\
\hline time-1.7 & 353 & 36 & 0.1 & 37 & 0.4 & 0.1 & $1(0.3 \%)$ \\
spell-1.0 & 475 & 63 & 0.1 & 64 & 0.8 & 0.1 & $1(0.2 \%)$ \\
barcode-0.96 & 1,729 & 322 & 1.1 & 335 & 5.7 & 1.0 & $5(0.3 \%)$ \\
archimedes & 2,467 & 423 & 5.0 & 1110 & 28.1 & 4.2 & $104(4.2 \%)$ \\
tar-1.13 & 5,244 & 301 & 7.4 & 469 & 316.1 & 8.9 & $75(1.4 \%)$ \\
\hline TOTAL & 10,268 & 1,145 & 13.7 & 2,015 & 351.1 & 14.3 & $186(1.8 \%)$ \\
\hline
\end{tabular}

Table 1. The minimal flow-sensitivity for interval abstract domain is significantly small. \#Var shows the number of program variables (abstract locations) in the programs. proved and time show the number of proved buffer-overrun queries in the programs and the running time of each analysis. Minimal flow-sensitivity proves exactly the same queries as the flow-sensitivity while taking analysis time comparable to that of flow-insensitivity.

able by 2-CFA analysis. We also observed that the conjecture holds for flow-sensitive numeric analysis and bufferoverrun queries. We implemented Liang and Naik's ACTIVECOARSEN algorithm, and found that the minimal flowsensitivity involves only $0.2-4.2 \%$ of total program variables, which means that we can achieve the precision of full flow-sensitivity with a cost comparable to that of flowinsensitivity (see Table 1).

\section{Learning via Bayesian optimisation}

We present our approach for learning a parameter of the adaptation strategy. We formulate the learning process as an optimisation problem, and solve it efficiently via Bayesian optimisation.

\subsection{The optimisation problem}

In our approach, learning a parameter from a codebase $\mathbf{P}=$ $\left\{P_{1}, \ldots, P_{m}\right\}$ corresponds to solving the following optimisation problem. Let $n$ be the number of features of our strategy in Section 4.1.

$$
\text { Find } \mathbf{w}^{*} \in \mathbb{R}^{n} \text { that maximises } \sum_{P_{i} \in \mathbf{P}} F\left(P_{i}, \mathcal{S}_{\mathbf{w}^{*}}\left(P_{i}\right)\right) \text {. }
$$

That is, the goal of the learning is to find $\mathrm{w}^{*}$ that maximises the number of proved queries on programs in $\mathbf{P}$ when these programs are analysed with the strategy $\mathcal{S}_{\mathbf{w}^{*}}$. However, solving this optimisation problem exactly is impossible. The objective function involves running static analysis $F$ over the entire codebase $\mathcal{S}_{\mathbf{w}^{*}}$ and is expensive to evaluate. Furthermore, it lacks a good structure-it is not convex and does not even have a derivative. Thus, we lower our aim slightly, and look for an approximate answer, i.e., a parameter $\mathbf{w}$ that makes the objective function close to its maximal value.

\subsection{Learning via random sampling}

A simple method for solving our problem in (5) approximately is to use random sampling (Algorithm 1). Although the method is simple and easy to implement, it is extremely inefficient according to our experience. In our experiments,

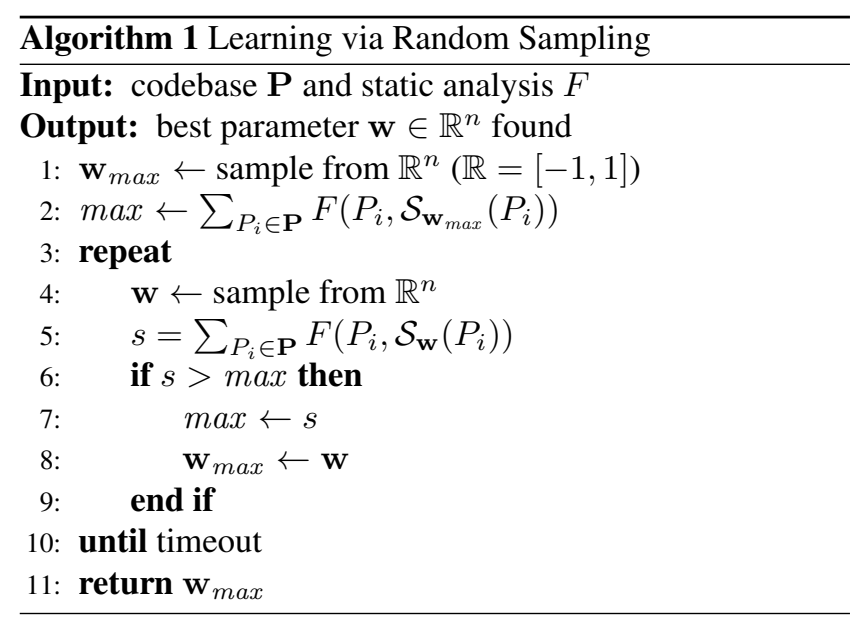

most of randomly sampled parameters have poor qualities, failing to prove the majority of queries on programs in $\mathbf{P}$ (Section 7.1). Thus, in order to find a good parameter using this method, we need to evaluate the objective function (running static analysis over the entire codebase) many times, but this is not feasible for realistic program analyses.

\subsection{Learning via Bayesian optimisation}

In this paper, we propose a better alternative for solving our optimisation problem in (5): use Bayesian optimisation for learning a good parameter of an adaptive static analyses. According to our experience, this alternative significantly outperforms the naive method based on random sampling (Section 7.1).

Bayesian optimisation is a powerful method for solving difficult optimisation problems where objective functions are expensive to evaluate [3] and do not have good structures, such as derivative. Typically, optimisers for such a problem work by evaluating its optimisation function with many different inputs and returning the input with the best output. The key idea of Bayesian optimisation is to reduce this number of evaluations by constructing and using a probabilistic model for the objective function. The model defines a probability distribution on functions, predicts what the ob- 


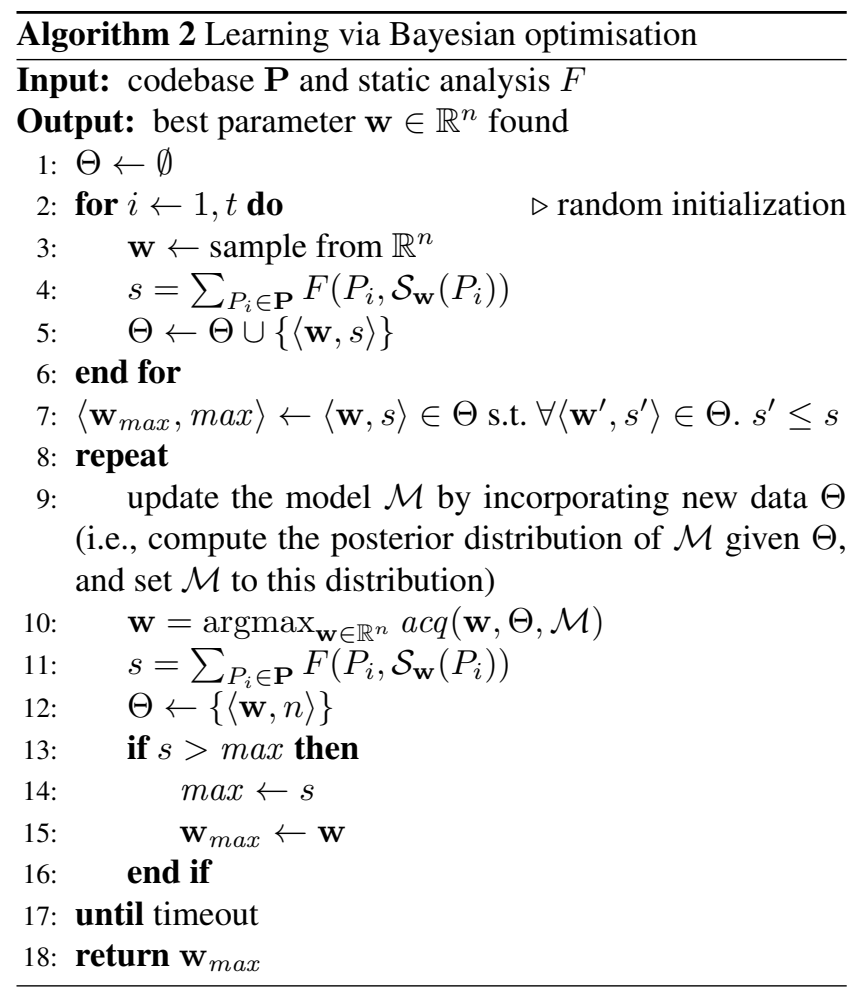

jective function looks like (i.e., mean of the distribution), and describes uncertainty on its prediction (i.e., variance of the distribution). The model gets updated constantly during the optimisation process (according to Bayes's rule), such that it incorporates the results of all the previous evaluations of the objective function. The purpose of the model is, of course, to help the optimiser pick a good input to evaluate next, good in the sense that the output of the evaluation is large and reduces uncertainty of the model considerably. We sum up our short introduction to Bayesian optimisation by repeating its two main components in our program-analysis application:

1. Probabilistic model $\mathcal{M}$ : Initially, this model $\mathcal{M}$ is set to capture a prior belief on properties of the objective function in (5), such as its smoothness. During the optimisation process, it gets updated to incorporate the information about all previous evaluations. ${ }^{5}$

2. Acquisition function $a c q$ : Given $\mathcal{M}$, this function gives each parameter $\mathbf{w}$ a score that reflects how good the parameter is. This is an easy-to-optimise function that serves as a proxy for our objective function in (5) when our optimiser chooses a next parameter to try. The function encodes a success measure on parameters $\mathbf{w}$ that balances two aims: evaluating our objective function with $\mathbf{w}$ should gives a large value (often called exploitation), and

\footnotetext{
${ }^{5}$ In the jargon of Bayesian optimisation or Bayesian statistics, the initial model is called a prior distribution, and its updated versions are called posterior distributions.
}

at the same time help us to refine our model $\mathcal{M}$ substantially (often called exploration).

Algorithm 2 shows our learning algorithm based on Bayesian optimisation. At lines 2-5, we first perform random sampling for $t$ times, and stores the pairs of parameter w and score $s$ in $\Theta$ (line 5). At line 7, we pick the best parameter and score in $\Theta$. The main loop is at lines from 8 to 17. At line 9 , we build the probabilistic model $\mathcal{M}$ from the collected data $\Theta$. At line 10, we select a parameter $\mathbf{w}$ by maximising the acquisition function. This takes some computation time but is insignificant compared to the cost of evaluating the expensive objective function (running static analysis over the entire codebase). Next, we run the static analysis with the selected parameter $\mathbf{w}$, and update the data (line 12). The loop repeats until we run out of our fixed time budget, at which point the algorithm returns the best parameter found.

Algorithm 2 leaves open the choice of a probabilistic model and an acquisition function, and its performance depends on making a right choice. We have found that a popular standard option for the model and the acquisition function works well for us-the algorithm with this choice outperforms the naive random sampling method substantially. Concretely, we used the Gaussian Process (GP) [21] for our probabilistic model, and the expected improvement (EI) [3] for the acquisition function.

A Gaussian Process (GP) is a well-known probabilistic model for functions to real numbers. In our setting, these functions are maps from parameters to reals, with the type $\mathbb{R}^{n} \rightarrow \mathbb{R}$. Also, a GP $F$ is a function-valued random variable such that for all $o \in \mathbb{N}$ and parameters $x \mathbf{w}_{1}, \ldots, \mathbf{w}_{o} \in \mathbb{R}^{n}$, the results of evaluating $F$ at these parameters

$$
\left\langle F\left(\mathbf{w}_{1}\right), \ldots, F\left(\mathbf{w}_{o}\right)\right\rangle
$$

are distributed according to the $o$-dimensional Gaussian distribution $^{6}$ with mean $\mu \in \mathbb{R}^{o}$ and covariance matrix $\boldsymbol{\Sigma} \in$ $\mathbb{R}^{o \times o}$, both of which are determined by two hyperparameters to the GP. The first hyperparameter is a mean function $m: \mathbb{R}^{n} \rightarrow \mathbb{R}$, and it determines the mean $\mu$ of the output of $F$ at each input parameter:

$$
\mu(\mathbf{w})=m(\mathbf{w}) \text { for all } \mathbf{w} \in \mathbb{R}^{n} .
$$

The second hyperparameter is a symmetric function $k$ : $\mathbb{R}^{n} \times \mathbb{R}^{n} \rightarrow \mathbb{R}$, called kernel, and it specifies the smoothness of $F$ : for each pair of parameters $\mathbf{w}$ and $\mathbf{w}^{\prime}, k\left(\mathbf{w}, \mathbf{w}^{\prime}\right)$ describes how close the outputs $F(\mathbf{w})$ and $F\left(\mathbf{w}^{\prime}\right)$ are. If $k\left(\mathbf{w}, \mathbf{w}^{\prime}\right)$ is positive and large, $F(\mathbf{w})$ and $F\left(\mathbf{w}^{\prime}\right)$ have similar values for most random choices of $F$. However, if

\footnotetext{
${ }^{6}$ A random variable $\mathbf{x}$ with value in $\mathbb{R}^{o}$ is a $o$-dimensional Gaussian random variable with mean $\mu \in \mathbb{R}^{o}$ and covariance matrix $\boldsymbol{\Sigma} \in \mathbb{R}^{o \times o}$ if it has the following probability density:
}

$$
p(\mathbf{x})=(2 \pi)^{-\frac{o}{2}} \times|\boldsymbol{\Sigma}|^{-\frac{1}{2}} \times \exp \left(-\frac{(\mathbf{x}-\mu)^{T} \boldsymbol{\Sigma}^{-1}(\mathbf{x}-\mu)}{2}\right)
$$


$k\left(\mathbf{w}, \mathbf{w}^{\prime}\right)$ is near zero, the values of $F(\mathbf{w})$ and $F\left(\mathbf{w}^{\prime}\right)$ do not exhibit such a close relationship. In our experiments, we adopted the common choice for hyperparameters and initialized a GP as follows:

$$
m=\lambda \mathbf{w} .0, \quad k\left(\mathbf{w}, \mathbf{w}^{\prime}\right)=\exp \left(-\left\|\mathbf{w}-\mathbf{w}^{\prime}\right\|^{2} / 2\right) .
$$

Incorporating data to the GP $F$ with $m$ and $k$ above is done by computing the so called posterior of $F$ with respect to the data. Suppose that we have evaluated our objective function with parameters $\mathbf{w}_{1}, \ldots, \mathbf{w}_{t}$ and obtained the values of the function $s_{1}, \ldots, s_{t}$. The value $s_{i}$ represents the number of proved queries when the static analysis is run with parameter $\mathbf{w}_{i}$ over the given codebase. Let $\Theta=\left\{\left\langle\mathbf{w}_{i}, s_{i}\right\rangle \mid 1 \leq i \leq n\right\}$. The posterior of $F$ with respect to $\Theta$ is a probability distribution obtained by updating the one for $F$ using information in $\Theta$. It is well-known that this posterior distribution $p(F \mid \Theta)$ is again a GP and has the following mean and kernel functions:

$$
\begin{aligned}
m^{\prime}(\mathbf{w}) & =\mathbf{k K}^{-1} \mathbf{s}^{T} \\
k^{\prime}\left(\mathbf{w}, \mathbf{w}^{\prime}\right) & =k\left(\mathbf{w}, \mathbf{w}^{\prime}\right)-\mathbf{k K}^{-1} \mathbf{k}^{\prime T}
\end{aligned}
$$

where

$$
\begin{aligned}
\mathbf{k}= & {\left[\begin{array}{llll}
k\left(\mathbf{w}, \mathbf{w}_{1}\right) & k\left(\mathbf{w}, \mathbf{w}_{2}\right) & \ldots & k\left(\mathbf{w}, \mathbf{w}_{t}\right)
\end{array}\right] } \\
\mathbf{k}^{\prime}= & {\left[\begin{array}{llll}
k\left(\mathbf{w}^{\prime}, \mathbf{w}_{1}\right) & k\left(\mathbf{w}^{\prime}, \mathbf{w}_{2}\right) & \ldots & k\left(\mathbf{w}^{\prime}, \mathbf{w}_{t}\right)
\end{array}\right] } \\
\mathbf{s}= & {\left[\begin{array}{llll}
s_{1} & s_{2} & \ldots & s_{t}
\end{array}\right] } \\
\mathbf{K}= & {\left[\begin{array}{ccc}
k\left(\mathbf{w}_{1}, \mathbf{w}_{1}\right) & \ldots & k\left(\mathbf{w}_{1}, \mathbf{w}_{t}\right) \\
\vdots & \ddots & \vdots \\
k\left(\mathbf{w}_{t}, \mathbf{w}_{1}\right) & \ldots & k\left(\mathbf{w}_{t}, \mathbf{w}_{t}\right)
\end{array}\right] }
\end{aligned}
$$

Figure 1 shows the outcomes of three posterior updates pictorially. It shows four regions that contain most of functions sampled from GPs.

The acquisition function for expected improvement (EI) is defined as follows:

$$
a c q(\mathbf{w}, \Theta, \mathcal{M})=E\left[\max \left(F(\mathbf{w})-s_{\max }, 0\right)\right] .
$$

Here $s_{\max }$ is the maximum score seen in the data $\Theta$ so far (i.e., $s_{\max }=\max \left\{s_{i} \mid \exists \mathbf{w}_{i} .\left\langle\mathbf{w}_{i}, s_{i}\right\rangle \in \Theta\right\}$ ), and $F$ is a random variable distributed according to the GP posterior with respect to $\Theta$ and is our model for the objective function. The formula $\max \left(F(\mathbf{w})-s_{\max }, 0\right)$ in the equation (6) measures the improvement in the maximum score when the objective function is evaluated at $\mathbf{w}$. The right hand side of the equation computes the expectation of this improvement, justifying the name "expected improvement". The further discussion on this acquisition function can be found in Section 2.3 of [3].

\section{Instances}

In this section, we present two instance analyses of our approach that adapt the degree of flow sensitivity and that of context-sensitivity, respectively.

\subsection{Partially flow-sensitive analysis}

We define a class of partially flow-sensitive analyses, and describe the features used in adaptation strategies for these analyses.

A class of partially flow-sensitive analyses Given a program $P$, let $(\mathbb{C}, \rightarrow)$ be its control flow graph, where $\mathbb{C}$ is the set of nodes (program points) and $(\rightarrow) \subseteq \mathbb{C} \times \mathbb{C}$ denotes the control flow relation of the program.

An analysis that we consider uses an abstract domain that maps program points to abstract states:

$$
\mathbb{D}=\mathbb{C} \rightarrow \mathbb{S} .
$$

Here an abstract state $s \in \mathbb{S}$ is a map from abstract locations (namely, program variables, structure fields and allocation sites) to values:

$$
\mathbb{S}=\mathbb{L} \rightarrow \mathbb{V} .
$$

For each program point $c$, the analysis comes with a function $f_{c}: \mathbb{S} \rightarrow \mathbb{S}$ that defines the abstract semantics of the command at $c$.

We assume that the analysis is formulated based on an extension of the sparse-analysis framework [20]. Before going into this formulation, let us recall the original framework for sparse analyses. Let $\mathrm{D}(c) \subseteq \mathbb{L}$ and $\mathrm{U}(c) \subseteq \mathbb{L}$ be the def and use sets at program point $c \in \mathbb{C}$. Using these sets, define a relation $(\rightsquigarrow) \subseteq \mathbb{C} \times \mathbb{L} \times \mathbb{C}$ for data dependency:

$$
\begin{aligned}
c_{0} \stackrel{l}{\rightsquigarrow} c_{n}= & \exists\left[c_{0}, c_{1}, \ldots, c_{n}\right] \in \text { Paths }, l \in \mathbb{L} \\
& l \in \mathrm{D}\left(c_{0}\right) \cap \mathrm{U}\left(c_{n}\right) \wedge \forall 0<i<n . l \notin \mathrm{D}\left(c_{i}\right)
\end{aligned}
$$

A way to read $c_{0} \stackrel{l}{\rightsquigarrow} c_{n}$ is that $c_{n}$ depends on $c_{0}$ on location $l$. This relationship holds when there exists a path $\left[c_{0}, c_{1}, \ldots, c_{n}\right]$ such that $l$ is defined at $c_{0}$ and used at $c_{n}$, but it is not re-defined at any of the intermediate points $c_{i}$. A sparse analysis is characterised by the following abstract transfer function $F: \mathbb{D} \rightarrow \mathbb{D}$ :

$$
F(X)=\lambda c . f_{c}\left(\lambda l \cdot \bigsqcup_{\substack{l \\ c_{0} \rightsquigarrow c}} X\left(c_{0}\right)(l)\right) .
$$

This analysis is fully sparse because it constructs data dependencies for every abstract location and tracks all these dependencies accurately.

We extend this sparse-analysis framework such that an analysis is allowed to track data dependencies only for abstract locations in some set $L \subseteq \mathbb{L}$, and to be flow-sensitive only for these locations. For the remaining locations (i.e., $\mathbb{L} \backslash L$ ), we use results from a quick flow-insensitive preanalysis [20], which we assume given. The results of this pre-analysis form a state $s_{I} \in \mathbb{S}$, and are stable (i.e., prefixpoint) at all program points:

$$
\forall c \in \mathbb{C} . f_{c}\left(s_{I}\right) \sqsubseteq s_{I}
$$


The starting point of our extension is to define the datadependency with respect to $L$ :

$$
\begin{aligned}
c_{0} \stackrel{l}{\rightsquigarrow} L c_{n}= & \exists\left[c_{0}, c_{1}, \ldots, c_{n}\right] \in \text { Paths, } l \in L . \\
& l \in \mathrm{D}\left(c_{0}\right) \cap \mathrm{U}\left(c_{n}\right) \wedge \forall 0<i<n . l \notin \mathrm{D}\left(c_{i}\right)
\end{aligned}
$$

The main modification lies in a new requirement that in order for $c_{0} \stackrel{l}{\rightsquigarrow} L c_{n}$ to hold, the location $l$ should be included in the set $L$. With this notion of data dependency, we next define an abstract transfer function:

$$
\begin{aligned}
& F_{L}(X)=\lambda c \cdot f_{c}\left(s^{\prime}\right) \\
& \text { where } s^{\prime}(l)=\left\{\begin{array}{lr}
X(c)(l) & (l \notin L) \\
\bigsqcup_{c_{0} \rightsquigarrow L c}^{l} X\left(c_{0}\right)(l) & \text { otherwise }
\end{array}\right.
\end{aligned}
$$

This definition says that when we collect an abstract state right before $c$, we use the flow-insensitive result $s_{I}(l)$ for a location not in $L$, and follow the original treatment for those in $L$. An analysis in our extension computes $\operatorname{Ifp}_{X_{0}} F_{L}$, where the initial $X_{0} \in \mathbb{D}$ is built by associating the results of the flow-insensitive analysis (i.e., values of $s_{I}$ ) with all locations not selected by $L$ (i.e., $\mathbb{L} \backslash L$ ):

$$
X_{0}(c)(l)=\left\{\begin{array}{lr}
s_{I}(l) & l \notin L \\
\perp & \text { otherwise }
\end{array}\right.
$$

Note that $L$ determines the degree of flow-sensitivity. For instance, when $L=\mathbb{L}$, the analysis becomes an ordinary flow-sensitive sparse analysis. On the other hand, when $L=$ $\emptyset$, the analysis is just a flow-insensitive analysis. The set $L$ is what we call abstraction in Section 3: abstraction locations in $\mathbb{L}$ form $\mathbb{J}_{P}$ in that section, and subsets of these locations, such as $L$, are abstractions there, which are expressed in terms of sets, rather than boolean functions. Our approach provides a parameterised strategy for selecting the set $L$ that makes the analysis comparable to the flow-sensitive version for precision and to the flow-insensitive one for performance. In particular, it gives a method for learning parameters in that strategy.

Features The features for our partially flow-sensitive analyses describe syntactic or semantic properties of abstract locations, namely, program variables, structure fields and allocation sites. Note that this is what our approach instructs, because these locations form the set $\mathbb{J}_{P}$ in Section 3 and are parts of $P$ where we control the precision of an analysis.

In our implementation, we used 45 features shown in Table 2, which describe how program variables, structure fields or allocation sites are used in typical $\mathrm{C}$ programs. When picking these features, we decided to focus on expressiveness, and included a large number of features, instead of trying to choose only important features. Our idea was to let our learning algorithm automatically find out such important ones among our features.

\begin{tabular}{|c|c|c|}
\hline Type & \# & Features \\
\hline \multirow[t]{33}{*}{ A } & 1 & local variable \\
\hline & 2 & global variable \\
\hline & 3 & structure field \\
\hline & 4 & location created by dynamic memory allocation \\
\hline & 5 & defined at one program point \\
\hline & 6 & location potentially generated in library code \\
\hline & 7 & assigned a constant expression (e.g., $\mathrm{x}=\mathrm{c} 1+\mathrm{c} 2$ ) \\
\hline & 8 & compared with a constant expression (e.g., $\mathrm{x}<\mathrm{c}$ ) \\
\hline & 9 & compared with an other variable (e.g., $\mathrm{x}<\mathrm{y}$ ) \\
\hline & 10 & negated in a conditional expression (e.g., if (!x)) \\
\hline & 11 & directly used in malloc (e.g., malloc(x)) \\
\hline & 12 & indirectly used in malloc (e.g., $y=x$; malloc $(y))$ \\
\hline & 13 & directly used in realloc (e.g., realloc(x)) \\
\hline & 14 & indirectly used in realloc (e.g., $y=x$; realloc $(y))$ \\
\hline & 15 & directly returned from malloc (e.g., $x=$ malloc(e)) \\
\hline & 16 & indirectly returned from malloc \\
\hline & 17 & directly returned from realloc (e.g., $x=$ realloc(e)) \\
\hline & 18 & indirectly returned from realloc \\
\hline & 19 & incremented by one (e.g., $x=x+1)$ \\
\hline & 20 & incremented by a constant expr. (e.g., $x=x+(1+2))$ \\
\hline & 21 & incremented by a variable (e.g., $x=x+y)$ \\
\hline & 22 & decremented by one (e.g., $\mathrm{x}=\mathrm{x}-1$ ) \\
\hline & 23 & decremented by a constant expr (e.g., $x=x-(1+2))$ \\
\hline & 24 & decremented by a variable (e.g., $x=x-y)$ \\
\hline & 25 & multiplied by a constant (e.g., $\mathrm{x}=\mathrm{x} * 2$ ) \\
\hline & 26 & multiplied by a variable (e.g., $x=x * y)$ \\
\hline & 27 & incremented pointer (e.g., p++) \\
\hline & 28 & used as an array index (e.g., a[x]) \\
\hline & 29 & used in an array expr. (e.g., x[e]) \\
\hline & 30 & returned from an unknown library function \\
\hline & 31 & modified inside a recursive function \\
\hline & 32 & modified inside a local loop \\
\hline & 33 & read inside a local loop \\
\hline \multirow[t]{12}{*}{ B } & 34 & $1 \wedge 8 \wedge(11 \vee 12)$ \\
\hline & 35 & $2 \wedge 8 \wedge(11 \vee 12)$ \\
\hline & 36 & $1 \wedge(11 \vee 12) \wedge(19 \vee 20)$ \\
\hline & 37 & $2 \wedge(11 \vee 12) \wedge(19 \vee 20)$ \\
\hline & 38 & $1 \wedge(11 \vee 12) \wedge(15 \vee 16)$ \\
\hline & 39 & $2 \wedge(11 \vee 12) \wedge(15 \vee 16)$ \\
\hline & 40 & $(11 \vee 12) \wedge 29$ \\
\hline & 41 & $(15 \vee 16) \wedge 29$ \\
\hline & 42 & $1 \wedge(19 \vee 20) \wedge 33$ \\
\hline & 43 & $2 \wedge(19 \vee 20) \wedge 33$ \\
\hline & 44 & $1 \wedge(19 \vee 20) \wedge \neg 33$ \\
\hline & 45 & $2 \wedge(19 \vee 20) \wedge \neg 33$ \\
\hline
\end{tabular}

Our features are grouped into Type A and Type B in the table. A feature of Type A describes a simple, atomic property for a program variable, a structure field or an allocation site, e.g., whether it is a local variable or not. A feature

Table 2. Features for partially flow-sensitive analysis. Features of Type A denote simple syntactic or semantic properties for abstract locations (that is, program variables, structure fields and allocation sites). Features of Type B are various combinations of simple features, and express patterns that variables are used in programs. 
of Type B, on the other hand, describes a slightly complex usage pattern, and is expressed as a combination of atomic features. Type B features have been designed by manually observing typical usage patterns of variables in the benchmark programs. For instance, feature 34 was developed after we observed the following usage pattern of variables:

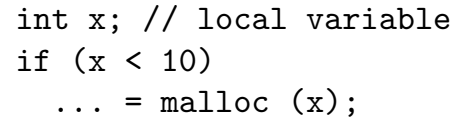

It says that $\mathrm{x}$ is a local variable, and gets compared with a constant and passed as an argument to a function that does memory allocation. Note that we included these Type B features not because they are important for flow-sensitivity. We included them to increase expressiveness, because our linear learning model with Type A features only cannot express such usage patterns. Deciding whether they are important for flow-sensitivity or not is the job of the learning algorithm.

\subsection{Partially context-sensitive analysis}

Another example of our approach is partially context-sensitive analyses. Assume we are given a program P. Let Procs be the set of procedures in $P$. The adaptation strategy of such an analysis selects a subset $\operatorname{Pr}$ of procedures of $P$, and instructs the analysis to treat only the ones in $\operatorname{Pr}$ context-sensitively: calling contexts of each procedure in $\operatorname{Pr}$ are treated separately by the analysis. This style of implementing partial context-sensitivity is intuitive and well-studied, so we omit the details and just mention that our implementation used one such analysis in [18] after minor modification. Note that these partially context-sensitive analyses are instances of the adaptive static analysis in Section 3; the set Procs corresponds to $\mathbb{J}_{P}$, and $\operatorname{Pr}$ is what we call an abstraction in that section.

For partial context-sensitivity, we used 38 features in Table 3 . Since our partially context-sensitive analysis adapts by selecting a subset of procedures, our features are predicates over procedures, i.e., $\pi^{k}:$ Procs $\rightarrow \mathbb{B}$. As in the flowsensitivity case, we used both atomic features (Type A) and compound features (Type B), both describing properties of procedures, e.g., whether a given procedure is a leaf in the call graph.

\subsection{Combination}

The previous two analyses can be combined to an adaptive analysis that controls both flow-sensitivity and contextsensitivity. The combined analysis adjusts the level of abstraction at abstract locations and procedures. This means that its $\mathbb{J}_{J}$ set consists of abstract locations and procedures, and its abstractions are just subsets of these locations and procedures. The features of the combined analysis are obtained similarly by putting together the features for our previous analyses. This combined abstractions and features enable our learning algorithm to find a more complex adaptation strategy that considers both flow-sensitivity and context-

\begin{tabular}{|c|l|l|}
\hline Type & $\#$ & \\
\hline A & 1 & leaf function \\
& 2 & function containing malloc \\
& 3 & function containing realloc \\
& 4 & function containing a loop \\
& 5 & function containing an if statement \\
& 6 & function containing a switch statement \\
& 7 & function using a string-related library function \\
& 8 & write to a global variable \\
& 9 & read a global variable \\
& 10 & write to a structure field \\
& 11 & read from a structure field \\
& 12 & directly return a constant expression \\
& 13 & indirectly return a constant expression \\
& 14 & directly return an allocated memory \\
& 15 & indirectly return an allocated memory \\
& 16 & directly return a reallocated memory \\
& 17 & indirectly return a reallocated memory \\
& 18 & return expression involves field access \\
& 19 & return value depends on a structure field \\
& 20 & return void \\
& 21 & directly invoked with a constant \\
& 22 & constant is passed to an argument \\
& 23 & invoked with an unknown value \\
& 24 & functions having no arguments \\
& 25 & functions having one argument \\
& 26 & functions having more than one argument \\
& 27 & functions having an integer argument \\
& 28 & functions having a pointer argument \\
& 29 & functions having a structure as an argument \\
\hline B & 30 & $2 \wedge(21 \vee 22) \wedge(14 \vee 15)$ \\
& 31 & $2 \wedge(21 \vee 22) \wedge \neg(14 \vee 15)$ \\
& 32 & $2 \wedge 23 \wedge(14 \vee 15)$ \\
& 33 & $2 \wedge 23 \wedge \neg(14 \vee 15)$ \\
34 & 35 & $2 \wedge(21 \vee 22) \wedge(16 \vee 17)$ \\
& 36 & $2 \wedge(21 \vee 22) \wedge \neg(16 \vee 17)$ \\
\hline
\end{tabular}

Table 3. Features for partially context-sensitive analysis.

sensitivity at the same time. This strategy helps the analysis to use its increased flexibility efficiently. In Section 7.2, we report our experience with experimenting the combined analysis.

\section{Experiments}

Following our recipe in Section 6, we instantiated our approach for partial flow-sensitivity and partial contextsensitivity, and implemented these instantiations in Sparrow, a buffer-overrun analysis for real-world C programs [19]. In this section, we report the results of our experiments with these implementations.

\subsection{Partial flow-sensitivity}

Setting We implemented a partial flow-sensitive analysis in Section 6.1 by modifying a buffer-overrun analyser for $\mathrm{C}$ 


\begin{tabular}{|c|c|c|c|c|c|c|c|c|c|c|c|c|c|}
\hline \multirow[b]{3}{*}{ Trial } & \multicolumn{4}{|c|}{ Training } & \multicolumn{9}{|c|}{ Testing } \\
\hline & \multirow{2}{*}{$\begin{array}{r}\text { FI } \\
\text { prove }\end{array}$} & \multirow{2}{*}{$\begin{array}{r}\text { FS } \\
\text { prove }\end{array}$} & \multicolumn{2}{|c|}{ partial FS } & \multicolumn{2}{|c|}{ FI } & \multicolumn{3}{|c|}{ FS } & \multicolumn{4}{|c|}{ partial FS } \\
\hline & & & prove & quality & prove & sec & prove & $\mathrm{sec}$ & cost & prove & sec & quality & $\cos t$ \\
\hline 1 & 6,383 & 7,316 & 7,089 & $75.7 \%$ & 2,788 & 48 & 4,009 & 627 & $13.2 \mathrm{x}$ & 3,692 & 78 & $74.0 \%$ & $1.6 \mathrm{x}$ \\
\hline 2 & 5,788 & 7,422 & 7,219 & $87.6 \%$ & 3,383 & 55 & 3,903 & 531 & $9.6 \mathrm{x}$ & 3,721 & 93 & $65.0 \%$ & $1.7 \mathrm{x}$ \\
\hline 3 & 6,148 & 7,842 & 7,595 & $85.4 \%$ & 3,023 & 49 & 3,483 & 1,898 & $38.6 \mathrm{x}$ & 3,303 & 99 & $60.9 \%$ & $2.0 \mathrm{x}$ \\
\hline 4 & 6,138 & 7,895 & 7,599 & $83.2 \%$ & 3,033 & 38 & 3,430 & 237 & $6.2 \mathrm{x}$ & 3,286 & 51 & $63.7 \%$ & $1.3 \mathrm{x}$ \\
\hline 5 & 7,343 & 9,150 & 8,868 & $84.4 \%$ & 1,828 & 28 & 2,175 & 577 & $20.5 \mathrm{x}$ & 2,103 & 54 & $79.3 \%$ & $1.9 \mathrm{x}$ \\
\hline TOTAL & 31,800 & 39,625 & 38,370 & $84.0 \%$ & 14,055 & 218 & 17,000 & 3,868 & $17.8 x$ & 16,105 & 374 & $69.6 \%$ & $1.7 x$ \\
\hline
\end{tabular}

Table 4. Effectiveness of our method for flow-sensitivity. prove: the number of proved queries in each analysis (FI: flowinsensitivity, FS: flow-sensitivity, partial FS: partial flow-sensitivity). quality: the ratio of proved queries among the queries that require flow-sensitivity. cost: cost increase compared to the FI analysis.

\begin{tabular}{|c|c|c|c|c|c|c|c|c|c|c|c|c|c|}
\hline \multirow[b]{3}{*}{ Trial } & \multicolumn{4}{|c|}{ Training } & \multicolumn{9}{|c|}{ Testing } \\
\hline & \multirow{2}{*}{$\begin{array}{r}\text { FICI } \\
\text { prove }\end{array}$} & \multirow{2}{*}{$\begin{array}{l}\text { FSCS } \\
\text { prove }\end{array}$} & \multicolumn{2}{|c|}{ partial FSCS } & \multicolumn{2}{|c|}{ FICI } & \multicolumn{3}{|c|}{ FSCS } & \multicolumn{4}{|c|}{ partial FSCS } \\
\hline & & & prove & quality & prove & $\mathrm{sec}$ & prove & $\mathrm{sec}$ & $\operatorname{cost}$ & prove & $\mathrm{sec}$ & quality & $\operatorname{cost}$ \\
\hline 1 & 6,383 & 9,237 & 8,674 & $80.3 \%$ & 2,788 & 46 & 4,275 & 5,425 & $118.2 \mathrm{x}$ & 3,907 & 187 & $75.3 \%$ & $4.1 \mathrm{x}$ \\
\hline 2 & 5,788 & 8,287 & 7,598 & $72.4 \%$ & 3,383 & 57 & 5,225 & 4,495 & $79.4 \mathrm{x}$ & 4,597 & 194 & $65.9 \%$ & $3.4 \mathrm{x}$ \\
\hline 3 & 6,148 & 8,737 & 8,123 & $76.3 \%$ & 3,023 & 48 & 4,775 & 5,235 & $108.8 \mathrm{x}$ & 4,419 & 150 & $79.7 \%$ & $3.1 \mathrm{x}$ \\
\hline 4 & 6,138 & 9,883 & 8,899 & $73.7 \%$ & 3,033 & 38 & 3,629 & 1,609 & $42.0 \mathrm{x}$ & 3,482 & 82 & $75.3 \%$ & $2.1 \mathrm{x}$ \\
\hline 5 & 7,343 & 10,082 & 10,040 & $98.5 \%$ & 1,828 & 30 & 2,670 & 7,801 & $258.3 \mathrm{x}$ & 2,513 & 104 & $81.4 \%$ & $3.4 \mathrm{x}$ \\
\hline TOTAL & 31,800 & 46,226 & 43,334 & $80.0 \%$ & 14,055 & 219 & 20,574 & 24,565 & $112.1 x$ & 18,918 & 717 & $74.6 \%$ & $3.3 x$ \\
\hline
\end{tabular}

Table 5. Effectiveness for Flow-sensitivity + Context-sensitivity.

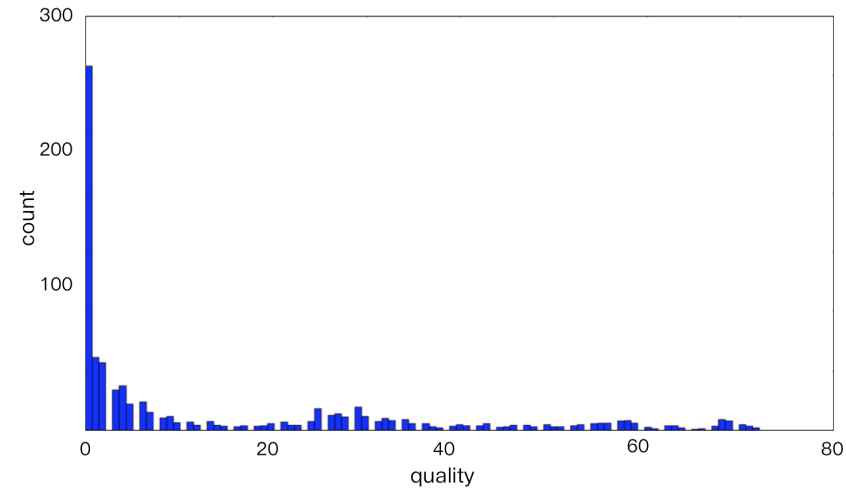

(a) Random sampling

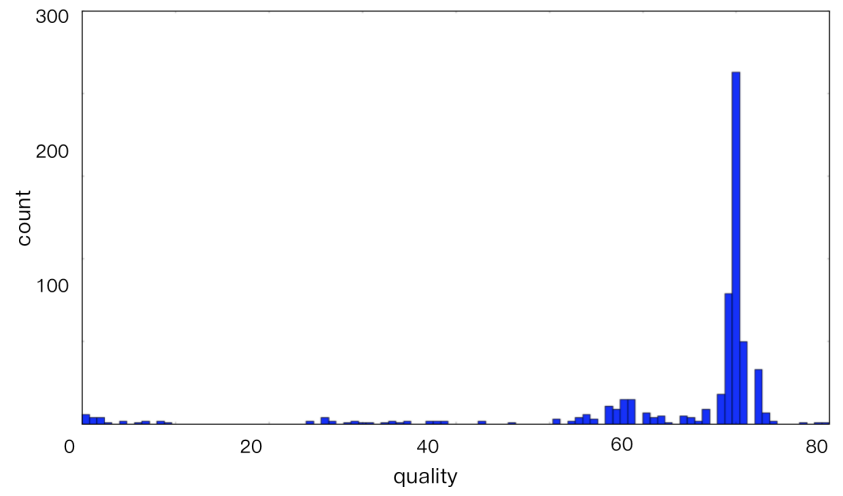

(b) Bayesian optimisation

Figure 2. Comparison of Bayesian optimisation with random sampling

programs, which supports the full $\mathrm{C}$ language and has been being developed for the past seven years [19]. This baseline analyser tracks both numeric and pointer-related information simultaneously in its fixpoint computation. For numeric values, it uses the interval abstract domain, and for pointer values, it uses an allocation-site-based heap abstraction. The analysis is field-sensitive (i.e., separates different structure fields) and flow-sensitive, but it is not context-sensitive. We applied the sparse analysis technique [20] to improve the scalability.

By modifying the baseline analyser, we implemented a partially flow-sensitive analyser, which controls its flowsensitivity according to a given set of abstract locations (pro- gram variables, structure fields and allocation sites) as described in Section 6.1. We also implemented our learning algorithm based on Bayesian optimisation. ${ }^{7}$ Our implementations were tested against 30 open source programs from GNU and Linux packages (Table 6 in Appendix).

The key questions that we would like to answer in our experiments are whether our learning algorithm produces a good adaptation strategy and how much it gets benefited from Bayesian optimisation. To answer the first question, we followed a standard method in the machine learning literature, called cross validation. We randomly divide the 30

\footnotetext{
${ }^{7}$ The implementation of our learning algorithm is available at http:// prl.korea.ac.kr/ hakjoo/research/oopsla15/.
} 
benchmark programs into 20 training programs and 10 test programs. An adaptation strategy is learned from the 20 training programs, and tested against the remaining 10 test programs. We repeated this experiment for five times. The results of each trial are shown in Table 4. In these experiments, we set $k=0.1$, which means that flow-sensitivity is applied to only the $10 \%$ of total abstract locations (i.e., program variables, structure fields and allocation sites). We compared the performance of a flow-insensitive analysis (FI), a fully flow-sensitive analysis (FS) and our partially flow-sensitive variant (partial FS). To answer the second question, we compared the performance of the Bayesian optimisation-based learning algorithm against the random sampling method.

Learning Table 4 shows the results of the training and test phases for all the five trials. In total, the flow-insensitive analysis (FI) proved 31,800 queries in the 20 training programs, while the fully flow-sensitive analysis (FS) proved 39,625 queries. During the learning phase, our algorithm found a parameter $w$. On the training programs, the analysis with w proved, on average, $84.0 \%$ of FS-only queries, that is, queries that were handled only by the flow-sensitive analysis (FS). Finding such a good parameter for training programs, let alone unseen test ones, is highly nontrivial. As shown in Table 2, the number of parameters to tune at the same time is $\mathbf{4 5}$ for flow-sensitivity. Manually searching for a good parameter $\mathrm{w}$ for these 45 parameter over 18 training programs is simply impossible. In fact, we tried to do this manual search in the early stage of this work, but most of our manual trials failed to find any useful parameter (Figure 2).

Figure 2 compares our learning algorithm based on Bayesian optimisation against the one based on random sampling. It shows the two distributions of the qualities of tried parameters $\mathbf{w}$ (recorded in the $x$ axis), where the first distribution uses parameters tried by random sampling over a fixed time budget (12h) and the second, by Bayesian optimisation over the same budget. By the quality of $\mathbf{w}$, we mean the percentage of FS-only queries proved by the analysis with $\mathbf{w}$. The results for random sampling (Figure 2(a)) confirm that the space for adaptation parameters $\mathrm{w}$ for partial flow-sensitivity is nontrivial; most of the parameters do not prove any queries. As a result, random sampling wastes most of its execution time by running the static analysis that does not prove any FS-only queries. This shortcoming is absent in Figure 2(b) for Bayesian optimisation. In fact, most parameters found by Bayesian optimisation led to adaptation strategies that prove about 70\% of FS-only queries. Figure 3 shows how the best qualities found by Bayesian optimisation and random sampling change as the learning proceeds. The results compare the first 30 evaluations for the first training set of our experiments, which show that Bayesian optimisation finds a better parameter $(63.5 \%)$ with fewer evaluations.

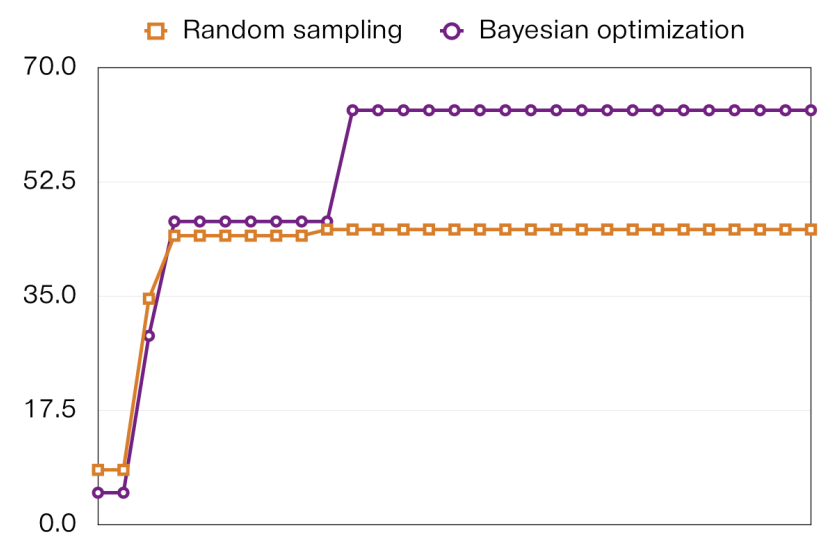

Figure 3. Comparison of Bayesian optimisation with random sampling

The random sampling method converged to the quality of $45.2 \%$.

Testing For each of the five trials, we tested a parameter learnt from 20 training programs, against 10 programs in the test set. The results of this test phase are given in Table 4 , and they show that the analysis with the learnt parameters has a good precision/cost balance. In total, for 10 test programs, the flow-insensitive analysis (FI) proved 14,055 queries, while the full flow-sensitive one (FS) proved 17,000 queries. The partially flow-sensitive version with a learnt adaptation strategy proved on average $69.6 \%$ of the FS-only queries. To do so, our partially flow-sensitive analysis increases the cost of the FI analysis only moderately (by $1.7 \mathrm{x}$ ), while the FS analysis increases the analysis cost by $17.8 \mathrm{x}$.

However, the results show that the analyses with the learnt parameters are generally less precise in the test set than the training set. For the five trials, our method has proved, on average, $84.0 \%$ of FS-queries in the training set and $69.6 \%$ in the test set.

Top-10 features The learnt parameter identified the features that are important for flow-sensitivity. Because our learning method computes the score of abstract locations based on linear combination of features and parameter $\mathbf{w}$, the learnt parameter $\mathbf{w}$ means the relative importance of features.

Figure 4 shows the 10 most important features identified by our learning algorithm from ten trials (including the five trials in Table 4 as well as additional five ones). For instance, in the first trial, we found that the most important features were \#19, 32, 1, 4, 28, 33, 29, 3, 43, 18 in Table 2 . These features say that accurately analysing, for instance, variables incremented by one (\#19) or modified inside a local loop (\#32), and local variables (\#1) are important for cost-effective flow-sensitive analysis. The histogram on the right shows the number of times each feature appears in the top-10 features during the ten trials. In all trials, features \#19 


\begin{tabular}{|c|c|c|c|c|c|c|c|c|c|c|}
\hline & \multicolumn{10}{|c|}{ Trials } \\
\cline { 2 - 9 } rank & 1 & 2 & 3 & 4 & 5 & 6 & 7 & 8 & 9 & 10 \\
\hline 1 & $\# 19$ & $\# 19$ & $\# 19$ & $\# 19$ & $\# 19$ & $\# 11$ & $\# 11$ & $\# 11$ & $\# 13$ & $\# 19$ \\
2 & $\# 32$ & $\# 32$ & $\# 32$ & $\# 32$ & $\# 32$ & $\# 19$ & $\# 19$ & $\# 19$ & $\# 19$ & $\# 28$ \\
3 & $\# 1$ & $\# 28$ & $\# 37$ & $\# 1$ & $\# 1$ & $\# 28$ & $\# 24$ & $\# 28$ & $\# 28$ & $\# 32$ \\
4 & $\# 4$ & $\# 33$ & $\# 40$ & $\# 27$ & $\# 4$ & $\# 12$ & $\# 26$ & $\# 12$ & $\# 32$ & $\# 7$ \\
5 & $\# 28$ & $\# 29$ & $\# 31$ & $\# 4$ & $\# 28$ & $\# 1$ & $\# 28$ & $\# 1$ & $\# 26$ & $\# 3$ \\
6 & $\# 33$ & $\# 18$ & $\# 1$ & $\# 28$ & $\# 7$ & $\# 32$ & $\# 32$ & $\# 4$ & $\# 7$ & $\# 33$ \\
7 & $\# 29$ & $\# 8$ & $\# 39$ & $\# 7$ & $\# 15$ & $\# 26$ & $\# 18$ & $\# 42$ & $\# 45$ & $\# 24$ \\
8 & $\# 3$ & $\# 14$ & $\# 27$ & $\# 9$ & $\# 33$ & $\# 21$ & $\# 43$ & $\# 23$ & $\# 3$ & $\# 20$ \\
9 & $\# 43$ & $\# 37$ & $\# 20$ & $\# 6$ & $\# 29$ & $\# 7$ & $\# 36$ & $\# 32$ & $\# 33$ & $\# 40$ \\
10 & $\# 18$ & $\# 9$ & $\# 4$ & $\# 15$ & $\# 3$ & $\# 45$ & $\# 7$ & $\# 6$ & $\# 35$ & $\# 8$ \\
\hline
\end{tabular}

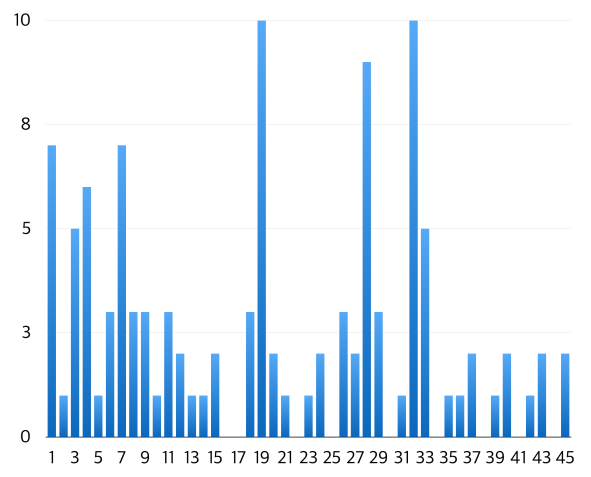

Figure 4. (Left) Top-10 features (for flow-sensitivity) identified by our learning algorithm for ten trials. Each entry denotes the feature numbers shown in Table 2. (Right) Counts of each feature (x-axis) that appears in the top-10 features during the ten trials. Features \#19 and \#32 are in top-10 for all trials. The results have been obtained with 20 training programs.

(variables incremented by one) and \#32 (variables modified inside a local loop) are included in the top-10 features. Features \#1 (local variables), \#4 (locations created by dynamic memory allocation), \#7 (location generated in library code), and \#28 (used as an array index) appear more than five times across the ten trials. We also identified top-10 features when trained with a smaller set of programs. Figure 5 shows the results with 10 training programs. In this case, features \#1 (local variables), \#7 (assigned a constant expression), \#9 (compared with another variable), \#19 (incremented by one), \#28 (used as an array index), and \#32 (modified inside a local loop) appeared more than five times across ten trials.

The automatically selected features generally coincided with our intuition on when and where flow-sensitivity helps. For instance, the following code (taken from barcode-0.96) shows a typical situation where flow-sensitivity is required:

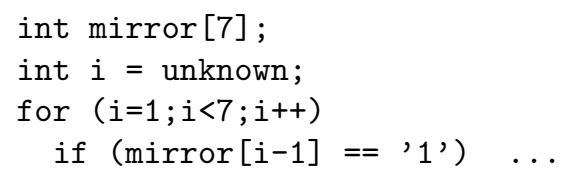

Because variable $i$ is initially unknown and is incremented in the loop, a flow-insensitive interval analysis cannot prove the safety of buffer access at line 3. On the other hand, if we analyze variable $i$ flow-sensitively, we can prove that $i-1$ at line 3 always has a value less than 7 (the size of mirror). Note that, according to the top-10 features, variable $i$ has a high score in our method because it is a local variable (\#1), modified inside a local loop (\#32), and incremented by one (\# 19).

The selected features also provided novel insights that contradicted our conjecture. When we manually identified important features in the early stage of this work, we conjectured that feature \#10 (variables negated in a conditional expression) would be a good indicator for flow-sensitivity, because we found the following pattern in the program under investigation (spell-1.0):

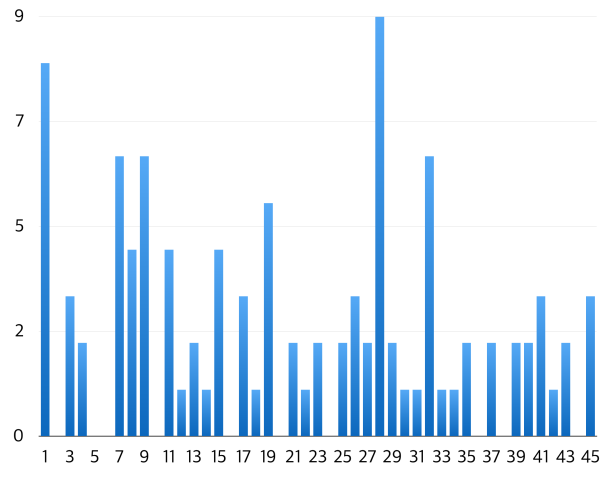

Figure 5. Top-10 feature frequencies with 10 training programs.

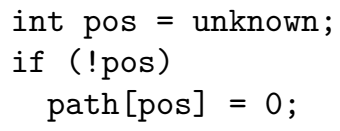

Although pos is unknown at line 1, its value at line 3 must be 0 because pos is negated in the condition at line 2 . However, after running our algorithm over the entire codebase, we found that this pattern happens only rarely in practice, and that feature \#10 is actually a strong indicator for flow"insensitivity".

Comparison with the impact pre-analysis approach [18] Recently $\mathrm{Oh}$ et al. proposed to run a cheap pre-analysis, called impact pre-analysis, and to use its results for deciding which parts of a given program should be analysed precisely by the main analysis [18]. We compared our approach with Oh et al.'s proposal on partial flow sensitivity. Following $\mathrm{Oh}$ et al.'s recipe [18], we implemented a impact pre-analysis that is fully flow-sensitive but uses a cheap abstract domain, in fact, the same one as in [18], which mainly tracks whether integer variables store non-negative values or not. Then, we built an analysis that uses the results of this pre-analysis for achieving partially flow-sensitivity. 
In our experiments with the programs in Table 4, the analysis based on the impact pre-analysis proved $80 \%$ of queries that require flow-sensitivity, and spent $5.5 \mathrm{x}$ more time than flow-insensitive analysis. Our new analysis of this paper, on the other hand, proved $70 \%$ and spent only $1.7 \mathrm{x}$ more time. Furthermore, in our approach, we can easily obtain the analysis that is selective both in flow- and contextsensitivity (Section 7.2), which is is nontrivial in the preanalysis approach.

\subsection{Adding partial context-sensitivity}

As another instance of our approach, we implemented an adaptive analysis for supporting both partial flow-sensitivity and partial context-sensitivity. Our implementation is an extension of the partially flow-sensitive analysis, and follows the recipe in Section 6.3. Its learning part finds a good parameter of a strategy that adapts flow-sensitivity and contextsensitivity simultaneously. This involves 83 parameters in total (45 for flow-sensitivity and 38 for context-sensitivity), and is a more difficult problem (as an optimisation problem as well as as a generalisation problem) than the one for partial flow-sensitivity only.

Setting We implemented context-sensitivity by inlining. All the procedures selected by our adaptation strategy get inlined. In order to avoid code explosion by such inlining, we inlined only relatively small procedures. Specifically, in order to be inlined in our experiments, a procedure should have less-than-100 basic blocks. The results of our experiments are shown in Table 6.3. In the table, FSCS means the fully flow-sensitive and fully context-sensitive analysis, where all procedures with less-than-100 basic blocks are inlined. FICI denotes the fully flow-insensitive and contextinsensitive analysis. Our analysis (partial FSCS) represents the analysis that selectively applies both flow-sensitivity and context-sensitivity.

Results The results show that our learning algorithm finds a good parameter $\mathbf{w}$ of our adaption strategy. The learnt w generalises well to unseen programs, and leads to an adaptation strategy that achieves high precision with reasonable additional cost. In training programs, FICI proved 26,904 queries, and FSCS proved 39,555 queries. With a learnt parameter $\mathbf{w}$ on training programs, our partial FSCS proved $79.3 \%$ of queries that require flow-sensitivity or context-sensitivity or both. More importantly, the parameter $\mathrm{w}$ worked well for test programs, and proved $81.2 \%$ of queries of similar kind. Regarding the cost, our partial FSCS analysis increased the cost of the FICI analysis only by $3.0 \mathrm{x}$, while the fully flow- and context-sensitive analysis (FSCS) increased it by $80.5 \mathrm{x}$.

\section{Related work and Discussion}

Parametric program analysis Parametric program analyses simply refer to a program analysis that is equipped with a class of program abstractions and analyses a given program by selecting abstractions from this class appropriately. Such analyses commonly adopt counter-example-guided abstraction refinement, and selects a program abstraction based on the feedback from a failed analysis run [1, 4-6, 8, 9, 31, 32]. Some exceptions to this common trend are to use the results of dynamic analysis [7, 16] or pre-analysis [18, 29] for finding a good program abstraction.

However, automatically finding such a strategy is not what they are concerned with, while it is the main goal of our work. All of the previous approaches focus on designing a good fixed strategy that chooses a right abstraction for a given program and a given query. A high-level idea of our work is to parameterise these adaptation (or abstractionselection) strategies, not just program abstractions, and to use an efficient learning algorithm (such as Bayesian optimisation) to find right parameters for the strategies. One interesting research direction is to try our idea with existing parametric program analyses.

For instance, our method can be combined with the impact pre-analysis [18] to find a better strategy for selective context-sensitivity. In [18], context-sensitivity is selectively applied by receiving a guidance from a pre-analysis. The pre-analysis is an approximation of the main analysis under full context-sensitivity. Therefore it estimates the impact of context-sensitivity on the main analysis, identifying context-sensitivity that is likely to benefit the final analysis precision. One feature of this approach is that the impact estimation of the pre-analysis is guaranteed to be realized at the main analysis (Proposition 1 in [18]). However, this impact realization does not guarantee the proof of queries; some context-sensitivity is inevitably applied even when the queries are not provable. Also, because the pre-analysis is approximated, the method may not apply context-sensitivity necessary to prove some queries. Our method can be used to reduce these cases; we can find a better strategy for selective context-sensitivity by using the pre-analysis result as a semantic feature together with other (syntactic/semantic) features for context-sensitivity.

Use of machine learning in program analysis Several machine learning techniques have been used for various problems in program analysis. Researchers noticed that many machine learning techniques share the same goal as program abstraction techniques, namely, to generalise from concrete cases, and they tried these machine learning techniques to obtain sophisticated candidate invariants or specifications from concrete test examples [17, 24-28]. Another application of machine learning techniques is to encode soft constraints about program specifications in terms of a probabilistic model, and to infer a highly likely specification of a given program by performing a probabilistic inference on the model $[2,11,13,22]$. In particular, Raychev et al.'s JSNice [22] uses a probabilistic model for describing type constraints and naming convention of JavaScript programs, 
which guides their cleaning process of messy JavaScript programs and is learnt from an existing codebase. Finally, machine learning techniques have also been used to mine correct API usage from a large codebase and to synthesize code snippets using such APIs automatically [14, 23].

Our aim is different from those of the above works. We aim to improve a program analysis using machine learning techniques, but our objective is not to find sophisticated invariants or specifications of a given program using these techniques. Rather it is to find a strategy for searching for such invariants. Notice that once this strategy is learnt automatically from an existing codebase, it is applied to multiple different programs. In the invariant-generation application, on the other hand, learning happens whenever a program is analysed. Our work identifies a new challenging optimisation problem related to learning such a strategy, and shows the benefits of Bayesian optimisation for solving this problem.

Application of Bayesian optimisation To the best of our knowledge, our work is the first application of Bayesian optimisation to static program analysis. Bayesian optimisation is a powerful optimisation technique that has been successfully applied to solve a wide range of problems such as automatic algorithm configuration [10], hyperparameter optimisation of machine learning algorithms [30], planning, sensor placement, and intelligent user interface [3]. In this work, we use Bayesian optimisation to find optimal parameters for adapting program analysis.

\section{Conclusion}

In this paper, we presented a novel approach for automatically learning a good strategy that adapts a static analysis to a given program. This strategy is learnt from an existing codebase efficiently via Bayesian optimisation, and it decides, for each program, which parts of the program should be treated with precise yet costly program-analysis techniques. This decision helps the analysis to strike a balance between cost and precision. Following our approach, we have implemented two variants of our buffer-overrun analyzer, that adapt the degree of flow-sensitivity and contextsensitivity of the analysis. Our experiments confirm the benefits of Bayesian optimisation for learning adaptation strategies. They also show that the strategies learnt by our approach are highly effective: the cost of our variant analyses is comparable to that of flow- and context-insensitive analyses, while their precision is close to that of fully flow- and context-sensitive analyses.

As we already mentioned, our learning algorithm is nothing but a method for generalizing information from given programs to unseen ones. We believe that this cross-program generalization has a great potential for addressing open challenges in program analysis research, especially because the amount of publicly available source code (such as that in GitHub) has increased substantially. We hope that our re- sults in this paper give one evidence of this potential and get program-analysis researchers interested in this promising research direction.

Acknowledgements This work was supported by the Engineering Research Center of Excellence Program of Korea Ministry of Science, ICT \& Future Planning(MSIP) / National Research Foundation of Korea(NRF) (Grant NRF2008-0062609), and by Samsung Electronics Software Center. This work was partly supported by Institute for Information \& communications Technology Promotion(IITP) grant funded by the Korea government(MSIP) (No. B0101-150557, Resilient Cyber-Physical Systems Research). Yang was supported by EPSRC.

\section{References}

[1] T. Ball and S. Rajamani. The SLAM project: Debugging system software via static analysis. In POPL, 2002.

[2] Nels E. Beckman and Aditya V. Nori. Probabilistic, modular and scalable inference of typestate specifications. In PLDI, pages 211-221, 2011.

[3] Eric Brochu, Vlad M. Cora, and Nando de Freitas. A tutorial on bayesian optimization of expensive cost functions, with application to active user modeling and hierarchical reinforcement learning. CoRR, abs/1012.2599, 2010.

[4] S. Chaki, E. Clarke, A. Groce, S. Jha, and H. Veith. Modular verification of software components in C. In ICSE, 2003.

[5] S. Grebenshchikov, A. Gupta, N. Lopes, C. Popeea, and A. Rybalchenko. HSF(C): A software verifier based on Horn clauses. In TACAS, 2012.

[6] B. Gulavani, S. Chakraborty, A. Nori, and S. Rajamani. Automatically refining abstract interpretations. In TACAS, 2008.

[7] Ashutosh Gupta, Rupak Majumdar, and Andrey Rybalchenko. From tests to proofs. STTT, 15(4):291-303, 2013.

[8] T. Henzinger, R. Jhala, R. Majumdar, and K. McMillan. Abstractions from proofs. In POPL, 2004.

[9] T. Henzinger, R. Jhala, R. Majumdar, and G. Sutre. Software verification with blast. In SPIN Workshop on Model Checking of Software, 2003.

[10] Frank Hutter, Holger H. Hoos, and Kevin Leyton-Brown. Sequential model-based optimization for general algorithm configuration. In Proceedings of the 5th International Conference on Learning and Intelligent Optimization, 2011.

[11] Ted Kremenek, Andrew Y. Ng, and Dawson R. Engler. A factor graph model for software bug finding. In IJCAI, pages 2510-2516, 2007.

[12] Percy Liang, Omer Tripp, and Mayur Naik. Learning minimal abstractions. In POPL, 2011.

[13] V. Benjamin Livshits, Aditya V. Nori, Sriram K. Rajamani, and Anindya Banerjee. Merlin: specification inference for explicit information flow problems. In PLDI, 2009.

[14] Alon Mishne, Sharon Shoham, and Eran Yahav. Typestatebased semantic code search over partial programs. In $O O P$ SLA, pages 997-1016, 2012. 
[15] Jonas Mockus. Application of bayesian approach to numerical methods of global and stochastic optimization. Journal of Global Optimization, 4(4), 1994.

[16] Mayur Naik, Hongseok Yang, Ghila Castelnuovo, and Mooly Sagiv. Abstractions from tests. In POPL, 2012.

[17] Aditya V. Nori and Rahul Sharma. Termination proofs from tests. In FSE, 2013.

[18] Hakjoo Oh, , Wonchan Lee, Kihong Heo, Hongseok Yang, and Kwangkeun Yi. Selective context-sensitivity guided by impact pre-analysis. In PLDI, 2014.

[19] Hakjoo Oh, Kihong Heo, Wonchan Lee, Woosuk Lee, and Kwangkeun Yi. Sparrow. http://ropas.snu.ac.kr/ sparrow.

[20] Hakjoo Oh, Kihong Heo, Wonchan Lee, Woosuk Lee, and Kwangkeun Yi. Design and implementation of sparse global analyses for C-like languages. In PLDI, 2012.

[21] Carl Edward Rasmussen and Christopher K. I. Williams. Gaussian Processes for Machine Learning (Adaptive Computation and Machine Learning). The MIT Press, 2005.

[22] Veselin Raychev, Martin Vechev, and Andreas Krause. Predicting program properties from "big code". In POPL, 2015.

[23] Veselin Raychev, Martin Vechev, and Eran Yahav. Code completion with statistical language models. In PLDI, 2014.

[24] Sriram Sankaranarayanan, Swarat Chaudhuri, Franjo Ivancic, and Aarti Gupta. Dynamic inference of likely data preconditions over predicates by tree learning. In ISSTA, 2008.

[25] Sriram Sankaranarayanan, Franjo Ivancic, and Aarti Gupta. Mining library specifications using inductive logic programming. In ICSE, 2008.

[26] Rahul Sharma, Saurabh Gupta, Bharath Hariharan, Alex Aiken, Percy Liang, and Aditya V. Nori. A data driven approach for algebraic loop invariants. In ESOP, 2013.

[27] Rahul Sharma, Saurabh Gupta, Bharath Hariharan, Alex Aiken, and Aditya V. Nori. Verification as learning geometric concepts. In $S A S, 2013$.

[28] Rahul Sharma, Aditya V. Nori, and Alex Aiken. Interpolants as classifiers. In $C A V, 2012$.

[29] Yannis Smaragdakis, George Kastrinis, and George Balatsouras. Introspective analysis: Context-sensitivity, across the board. In PLDI, 2014.

[30] Jasper Snoek, Hugo Larochelle, and Ryan P. Adams. Practical bayesian optimization of machine learning algorithms. In 26th Annual Conference on Neural Information Processing Systems, 2012.

[31] Xin Zhang, Ravi Mangal, Radu Grigore, Mayur Naik, and Hongseok Yang. On abstraction refinement for program analyses in datalog. In PLDI, 2014.

[32] Xin Zhang, Mayur Naik, and Hongseok Yang. Finding optimum abstractions in parametric dataflow analysis. In PLDI, 2013.

\begin{tabular}{|l|r|}
\hline Programs & LOC \\
\hline cd-discid-1.1 & 421 \\
time-1.7 & 1,759 \\
unhtml-2.3.9 & 2,057 \\
spell-1.0 & 2,284 \\
mp3rename-0.6 & 2,466 \\
ncompress-4.2.4 & 2,840 \\
pgdbf-0.5.0 & 3,135 \\
cam-1.05 & 5,459 \\
e2ps-4.34 & 6,222 \\
sbm-0.0.4 & 6,502 \\
mpegdemux-0.1.3 & 7,783 \\
barcode-0.96 & 7,901 \\
bzip2 & 9,796 \\
bc-1.06 & 16,528 \\
gzip-1.2.4a & 18,364 \\
unrtf-0.19.3 & 19,015 \\
archimedes & 19,552 \\
coan-4.2.2 & 28,280 \\
gnuchess-5.05 & 28,853 \\
tar-1.13 & 30,154 \\
tmndec-3.2.0 & 31,890 \\
agedu-8642 & 32,637 \\
gbsplay-0.0.91 & 34,002 \\
flake-0.11 & 35,951 \\
enscript-1.6.5 & 38,787 \\
mp3c-0.29 & 52,620 \\
tree-puzzle-5.2 & 62,302 \\
icecast-server-1.3.12 & 68,564 \\
aalib-1.4p5 & 73,412 \\
rnv-1.7.10 & 93,858 \\
\hline TOTAL & 743,394 \\
\hline
\end{tabular}

Table 6. Benchmark programs.

\section{A. Benchmarks}

Table 6 presents the list of 30 benchmark programs used in our experiments. The benchmark programs are mostly from GNU and Linux packages. 